\title{
Metabolic surgery in treatment of obese Japanese patients with type 2 diabetes: a joint consensus statement from the Japanese Society for Treatment of Obesity, the Japan Diabetes Society, and the Japan Society for the Study of Obesity
}

\author{
Akira Sasaki ${ }^{1}$ - Koutaro Yokote ${ }^{2} \cdot$ Takeshi Naitoh $^{3} \cdot$ Junji Fujikura $^{4} \cdot$ Karin Hayashi $^{5} \cdot$ Yushi Hirota $^{6}$. \\ Nobuya Inagaki ${ }^{4} \cdot$ Yasushi Ishigaki $^{7} \cdot \mathrm{Kazunori} \mathrm{Kasama}^{8} \cdot$ Eri Kikkawa $^{8} \cdot \operatorname{Hidenori~Koyama~}^{9} \cdot$ Hiroaki Masuzaki $^{10}$. \\ Takeshi Miyatsuka ${ }^{11}$. Takehiro Nozaki ${ }^{12}$. Wataru Ogawa ${ }^{6} \cdot$ Masayuki Ohta $^{13}$. Shinichi Okazumi ${ }^{14}$. \\ Michio Shimabukuro ${ }^{15}$. lichiro Shimomura ${ }^{16} \cdot$ Hitoshi Nishizawa $^{16}$. Atsuhito Saiki ${ }^{17}$. Yosuke Seki ${ }^{8}$. \\ Nobuhiro Shojima ${ }^{18} \cdot$ Motoyoshi Tsujino $^{19} \cdot$ Satoshi Ugi ${ }^{20} \cdot$ Hiroaki Watada ${ }^{11} \cdot$ Toshimasa Yamauchi $^{18}$. \\ Takashi Yamaguchi $^{17} \cdot$ Kohjiro Ueki $^{21} \cdot$ Takashi Kadowaki $^{22}$ • Ichiro Tatsuno ${ }^{23}$. Joint Committee in the Japanese \\ Society for Treatment of Obesity, the Japan Diabetes Society, the Japan Society for the Study of Obesity
}

Received: 6 October 2021/ Published online: 8 November 2021

(c) The Japan Diabetes Society 2021, corrected publication 2021

\begin{abstract}
Bariatric surgery has been shown to have a variety of metabolically beneficial effects for patients with type 2 diabetes (T2D), and is now also called metabolic surgery. At the 2nd Diabetes Surgery Summit held in 2015 in London, the indication for bariatric and metabolic surgery was included in the "algorithm for patients with type T2D". With this background, the Japanese Society for Treatment of Obesity (JSTO), the Japan Diabetes Society (JDS) and the Japan Society for the Study of Obesity (JASSO) have formed a joint committee to develop a consensus statement regarding bariatric and metabolic surgery for the treatment of Japanese patients with T2D. Eventually, the consensus statement was announced at the joint meeting of the 38th Annual Meeting of JSTO and the 41st Annual Meeting of JASSO convened in Toyama on March 21, 2021. In preparing the consensus statement, we used Japanese data as much as possible as scientific evidence to consider the indication criteria, and set two types of recommendation grades, "recommendation" and "consideration", for items for which recommendations are possible. We hope that this statement will be helpful in providing evidence-based high-quality care through bariatric and metabolic surgery for the treatment of obese Japanese patients with T2D.
\end{abstract}

Keywords Obesity $\cdot$ Diabetes $\cdot$ Metabolic surgery $\cdot$ Bariatric surgery $\cdot$ Sleeve gastrectomy $\cdot$ Duodenojejunal bypass

\section{Introduction}

Overweight and obesity are defined as abnormal or excessive fat accumulation, and are associated with an increased risk of obesity-related comorbidities such as type 2 diabetes (T2D), dyslipidemia, hypertension and sleep apnea syndrome. The World Health Organization reported that the prevalence of obesity has tripled from 1975 [1]. Since Japanese people tend to be at risk of obesity-related comorbidities such as T2D even when they are mildly overweight,

Akira Sasaki

sakira@iwate-med.ac.jp

Extended author information available on the last page of the article obesity has been defined as body mass index (BMI) $\geq 25 \mathrm{~kg} /$ $\mathrm{m}^{2}$ in Japan, and Japan was the first to introduce the concept of "obesity disease", which are potentially involved in the onset or worsening of illness and require weight control [2].

Bariatric surgery was initially developed for the treatment of severely obese patients with BMI $35 \mathrm{~kg} / \mathrm{m}^{2}$ or higher, and the efficacy and safety in this patient population have been proven [3-5]. In recent years, bariatric surgery for patients with T2D has been shown to have a variety of metabolically beneficial effects including remission of T2D, independently of weight loss, and thus is now also called metabolic surgery. In Europe and the United States, bariatric/metabolic surgery has been recognized as one of the treatments for T2D. At the 2nd Diabetes Surgery Summit held in 2015 in London, the 
indication for bariatric and metabolic surgery was included in the "algorithm for patients with type T2D" [6], and was endorsed by the Japanese Society for Treatment of Obesity (JSTO) and the Japan Diabetes Society (JDS).

With this background, JSTO, JDS and the Japan Society for the Study of Obesity (JASSO) decided to form a joint committee to develop a consensus statement regarding bariatric and metabolic surgery for the treatment of Japanese patients with T2D based on Japanese evidence. The first joint committee was held in Tokyo on November 3, 2019 , and the final draft was completed after six sessions of deliberation by the committee. The consensus statement was announced at the joint meeting of the 38th Annual Meeting of JSTO and the 41st Annual Meeting of JASSO convened in Toyama on March 21, 2021. This paper describes the details of the consensus statement. We hope that this statement will be helpful in providing evidence-based high-quality care through bariatric and metabolic surgery for the treatment of obese Japanese patients with T2D.

\section{Treatment for obesity}

\section{Current status and thoughts behind the diagnosis and treatment for obesity and obesity-associated T2D}

Medical care for obesity in Japan clearly distinguishes between obesity, defined by BMI and obesity disease (obesity) requiring the patient to lose weight from a medical perspective (Fig. 1) [7]. A patient with a BMI of $\geq 35 \mathrm{~kg} / \mathrm{m}^{2}$ is classified as severely obese and is diagnosed was severe obesity if the patient also has a health disorder caused by or related to obesity. Severe obesity involves significant health disorders and is, in some cases, refractory. Diet, exercise, and cognitive-behavioral therapy are important medical options for obesity. For severe obesity, surgical and medical treatments including pharmacotherapy, diet, exercise, and cognitive-behavioral therapy are therapeutic options. Because obesity causes diverse health disorders, including diabetes and diabetic complications, heart failure, renal failure, and cancer, the interdisciplinary cooperation of medical professionals from various specialties is required.

In obese patients with $\mathrm{T} 2 \mathrm{D}$, in addition to the possibility of secondary obesity should be excluded, it is essential to investigate their lifestyle and psychological factors and help improve and reduce the inappropriate lifestyle habits that contribute to obesity $[8,9]$.

The purpose of diet therapy for obesity with T2D is to prevent complications and restrain disease progression by maintaining a generally favorable metabolic state. Therefore, while patients have their total caloric intake set according to their body weight, it is necessary to personalize the target body weight by considering that it can vary according to factors such as age and disease severity. First, the target total caloric intake level is decided at the beginning treatment. It is later modified considering the patient's pathology, age, body composition, and metabolic state, adherence and changes of the body weight. The target body weight $(\mathrm{kg})$ is calculated using the formula below by considering that the BMI with the lowest number of total deaths is different by age and that there is a certain range to the body weight. The target body weight $(\mathrm{kg})$ is calculated using the formula below by considering that the BMI with the lowest number

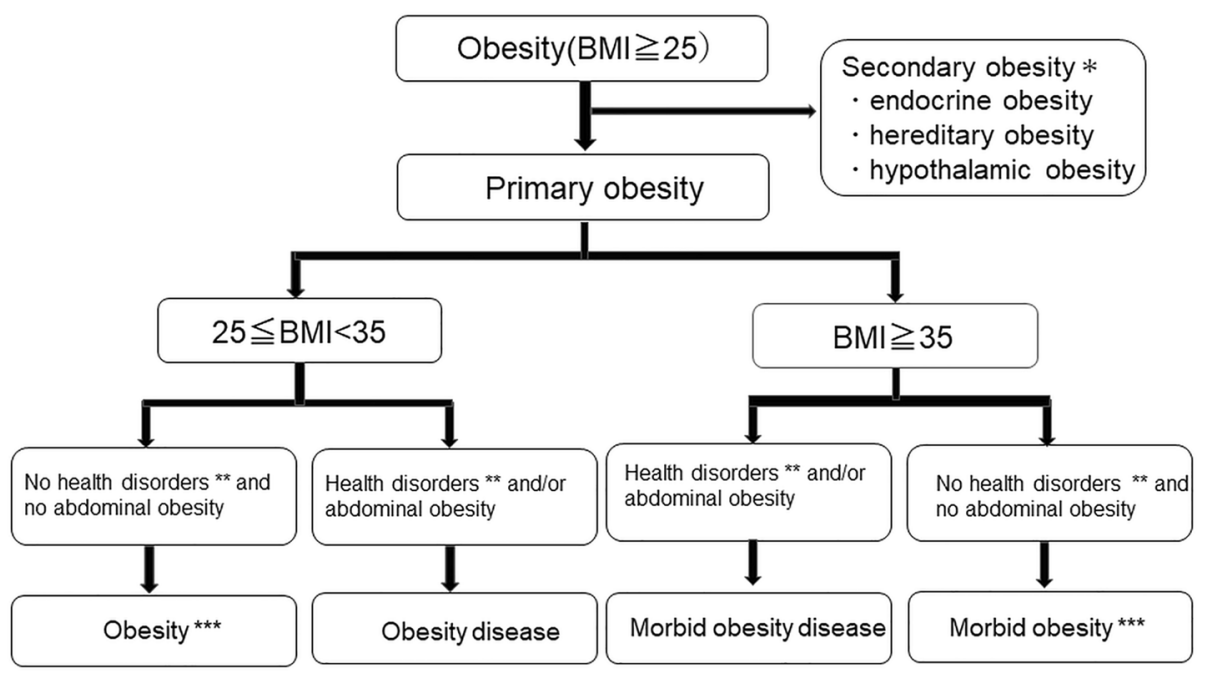

- Always consider possibility ** See Table on health problems *** Weightmanagement is essential to obesity and morbid obesity

Fig. 1 Diagnostic flowchart for obesity disease 
of total deaths differs by age and that the target weight has a certain range.

\section{Target weight $\mathbf{( k g )}$}

Age $<65$ years old: $[\text { Height }(\mathrm{m})]^{2} \times 22$.

Age 65-74 years old: [Height $(\mathrm{m})]^{2} \times 22 \sim 25$.

Age $\geq 75$ years old: [Height $(\mathrm{m})]^{2} \times 22 \sim 25^{*}$.

*In the latter half of the elderly age group (age $\geq 75$ years old), based on the current weight, it should be determined based on the assessment of frailty, complications, body composition, shortening of height, eating status and metabolic status.

\section{Energy coefficients ( $\mathrm{kcal} / \mathbf{k g}$ ) according to physical activity level and pathology}

Light exertion (mostly sedentary static activity): 25-30.

Normal exertion (mostly sedentary but includes commuting, housework, and light exercise): 30-35

Heavy exertion (strenuous work, vigorous exercise habits): $35 \sim$

In the case of obesity and weight loss, a coefficient smaller than the physical activity level can be set. If there is a large discrepancy between the target weight and the current weight, the coefficient should be set flexibly.

\section{Guideline for total energy intake}

Total energy intake $(\mathrm{kcal} /$ day $)=$ Target body weight $(\mathrm{kg}) \mathrm{x}$ Energy coefficient $(\mathrm{kcal} / \mathrm{kg})$.

A possible low-calorie diet for patients with severe obesity provides a target body weight of $20-25 \mathrm{kcal} / \mathrm{kg}$. A very low-calorie diet ( $600 \mathrm{kcal} / \mathrm{day}) \mathrm{can}$ be considered for inpatient with severe obesity if the physical and psychological symptoms are strictly observed and water intake is adequately management. To maintain the body's protein level, diet therapy must provide sufficient proteins containing essential amino acids, vitamins, and minerals [7].

Exercise therapy is one of the basic treatments for obesity and diabetes, allowing patients to lose weight via increased energy consumption. Patients must be assessed for complications such as cardiovascular diseases before initiating exercise therapy. The frequency, intensity, duration, and type of exercise are selected by prioritizing the patient's efficacy and safety $[7,8]$.

Cognitive-behavioral therapy is effective for patients with obesity to lose weight and maintain the reduced weight [10, 11]. Since abnormal eating behavior such as fast eating and snacking is often seen in patients with obesity, it can be useful to evaluate a patient's dietary behavior by measuring body weight, revising the nutritional records, and motivating them to stick to the right dietary regimen regularly.
Pharmacotherapy is considered when a patient's obesity fails to improve adequately despite receiving guidance on improving lifestyle habits for a certain period of time. Mazindol, which has an appetite-suppressing effect, can be prescribed to patients with severe obesity with a BMI of $\geq 35 \mathrm{~kg} / \mathrm{m}^{2}$ or an obesity degree of $\geq 70 \%$. It can be used continuously for up to 3 months, and a 14-day prescription is given each time. It is contraindicated for patients with psychiatric disorders such as anxiety, depression, and schizophrenia or patients with a history of drug and alcohol abuse, cerebrovascular diseases, and other conditions. If prescribed, close monitoring for pulmonary hypertension (one of the representative adverse) should be done. In patients with obesity and T2D, weight loss and improved blood glucose levels may be achieved using drugs such as using glucagon-like peptide-1 (GLP-1) receptor agonists and sodium-glucose co-transporter 2 (SGLT2) inhibitors [12-15].

Coronavirus disease has been spreading worldwide. It has come to light that COVID-19 aggravation is closely related to obesity and diabetes [16, 17]. Because as COVID-19 tends to become severe when a patient's blood glucose level is high, it is important to diagnose the infection early and treat it appropriately before it becomes severe. Even in the midst of the COVID-19 pandemic, patients should regularly exercise indoors and away from crowds and consult a physician regularly to monitor blood glucose levels and body weight and have the test results reviewed.

\section{Significance of the ideal metabolic surgery in comprehensive treatment for obesity}

1.1. Metabolic surgery has a weight-loss effect and metabolically beneficial effects and is effective treatment for severe obesity patients with difficulty in losing weight and T2D patients with obesity if an appropriate support system and safety are ensured before and after the surgery. (recommendation for severe obesity, consideration for obesity).

A meta-analysis involving studies in East Asians reported that metabolic surgery for obesity resulted in weight loss and improved complications 3-5 years after surgery [18]. In a meta-analysis of randomized controlled trials, which included studies involving East Asians, the laparoscopic Roux-en-Y gastric bypass (LRYGB) group had a weight loss of $6.54 \mathrm{~kg} / \mathrm{m}^{2}$ by BMI and reduced cardiovascular risk compared to the non-surgical medical treatment group in patients with obesity and T2D [19]. A meta-analysis of randomized controlled trials and observational trials, including studies involving East Asians, reported that metabolic surgery for obesity led to weight loss and improvement in T2D [20, 21]. 
Laparoscopic sleeve gastrectomy (LSG) is covered by insurance and is becoming more common in Japan. In addition to weight loss, metabolic surgery favors glucose metabolism such as improvement of insulin resistance and insulin secretion in patients with T2D. It is also effective for diabetes with advanced complications and long disease duration, but its metabolism-improving effect is greater for diabetes of short disease duration. It is important to consider metabolic surgery according to each patient's diabetes pathology and lifestyle and by referring to a consensus statement. Metabolic surgery requires a preoperative assessment of complications and approval for proceeding with surgery by a multidisciplinary medical team, consisting of physicians, nurses, registered dietitians, pharmacists, physiotherapists, and certified psychologists, and it requires the establishment of a healthcare system for lifelong follow-up of nutritional disorders and mental health after surgery [22].

When treating obesity and T2D, the multidisciplinary medical team needs to share information on parameters such as the patient's lifestyle habits and agree on the selected course of therapeutic action. Close cooperation among staff is indispensable for understanding the patient's condition and providing forms of support that respect the patient's lifestyle. Even with a dedicated team involved, it can take time for a patient's behavior to change. Thus, it is important to continue providing an environment in which the patient feels safe to consult. Studies have indicated that around half of patients with severe obesity who require metabolic surgery have psychiatric comorbidities such as depression [23]. For such patients, it is necessary to consider collaborating interventions with psychiatrists and clinical psychologists. Improving the quality of therapeutic guidance and increasing number of the personnel enable multidisciplinary medical teams to provide detailed support to each patient.

\section{Importance of advocacy for patients with obesity or T2D and obesity}

Due to popular misunderstandings and prejudice concerning obesity and severe obesity (e.g., the brief that they result from a lack of self-responsibility), many false perceptions of the disease have been spread [24]. Patients with obesity and severe obesity may suffer from social stigma that is discrimination due to social prejudice. Due to the misconception that simply eating less and increasing exercise can cure obesity completely, patients with obesity in the workplace, schools, and society suffer from baseless and deep-seated prejudice according to which they are sloppy, gluttonous, and incapable of taking care of themselves. To effectively prevent and treat obesity and make further advancements in obesity research, healthcare professionals, researchers, media professionals, policymakers, and patients should work together to disseminate new and correct information based on scientific knowledge. There is a gap in awareness about obesity between patients and healthcare professionals that needs to be closed [25]. Around $81 \%$ of patients think that losing weight is their responsibility, and approximately $30 \%$ of medical staff believe it is patients' responsibility to lose weight. Around $7 \%$ patients express no interest in losing weight, but approximately $71 \%$ of medical staff believe patients have no interest in losing weight.

Activities supporting a specific group or initiative are called advocacies. Promoting advocative activities to create social awareness about obesity and severe obesity is the key to increasing therapeutic opportunities for patients and controlling disease aggravation. Advocative activities at the personal and hospital levels and at regional, national, and international levels rather than efforts only by academic societies and institutions, play a helpful role in a eliminating the stigma related to obesity (known as obesity stigma). In addition to the social stigma to which patients are subjected, obesity stigma involves the self-inflicted stigma that become aware of the social stigma [24]. Gathering in Kobe, where the 39th Conference of the JASSO, the 23 academic societies related to obesity that are part of the Japanese Medical Science Federation agreed to collaborate across domains to eradicate obesity, proposing the Kobe Declaration 2018.

Despite improvements resulting from diabetes treatments, such as reasonable blood sugar control and quality of life similar to that of a healthy person, there have been reports of patients who lack access to the necessary services, thus placing them at a disadvantage by influencing factors such as their employment and promotion [26]. If such stigma is left unattended, patients tend to conceal their diabetes, miss opportunities to receive proper treatment, and later suffer from aggravation and complications of diabetes. The Japan Diabetes Society and the Japan Association for Diabetes Education and Care recognize the seriousness of the social stigma surrounding diabetes patients. They now strive to create a society free of such stigma to prevent diabetes patients from hiding their disease.

Because body constitution is highly involved in the onset of obesity, it is necessary to elucidate the physiological constitution characteristics of Japanese and Asian people. In a whole-genome analysis of 339,224 Westerners, 97 BMIrelated genes were identified, and pathway analysis showed significant involvement in the central nervous system [27]. The whole-genome analysis of 173,430 Japanese individuals led to the identification of 112 BMI-related genes. In contrast, pathway analysis identified the participation of the central nervous system, B lymphocytes, and adipose tissue [28]. In a whole-genome analysis of 433,540 Asian individuals, 61 new T2D genes were identified. Pathway analysis has identified genes that reduce the energy storage capacity of subcutaneous fat and cause visceral fat accumulation [29]. 


\section{Requirements for surgical introduction and surgical indication criteria}

\section{Indication criteria of metabolic surgery for T2D}

2.1. Metabolic surgery is recommended as a treatment option regardless of glycemic control if the patient has T2D with BMI $\geq 35 \mathrm{~kg} / \mathrm{m}^{2}$ at the time of consultation and the BMI $\geq 35 \mathrm{~kg} / \mathrm{m}^{2}$ persists despite treatments by a diabetologist or obesity specialist for $\geq 6$ months. (recommendation)

2.2. Metabolic surgery should be considered a treatment option if the patient has T2D with BMI $\geq 32 \mathrm{~kg} / \mathrm{m}^{2}$ at the time of consultation and has not achieved $\geq 5 \%$ weight loss or has achieved it but continues to have poor glycemic control (HbA1c $>8.0 \%)$ despite treatments by a diabetologist or obesity specialist for $\geq 6$ months. (consideration)

\section{Global indications of metabolic surgery}

According to the consensus statements of the International Federation for the Surgery of Obesity and Metabolic Disorders (IFSO)-Asia Pacific Chapter, bariatric and gastrointestinal metabolic surgery should be considered for Asian patients with BMI $\geq 30 \mathrm{~kg} / \mathrm{m}^{2}$ who have T2D or metabolic syndrome that is poorly controlled by non-surgical medical treatments [30]. Bariatric surgery has been observed to improve glucose metabolism early on after surgery, even before weight loss occurs. It was found that the mechanism of this improvement is not only reduced caloric intake but also changes in various gastrointestinal hormones. Given this background, bariatric surgery has come to be recognized as a useful therapeutic method that can be expected to have a weight-loss effect and a metabolism-improving effect, and is generally called metabolic surgery [6]. The Second Diabetes Surgery Summit announced a treatment algorithm that recommends metabolic surgery for Asian patients with BMI of $\geq 37.5 \mathrm{~kg} / \mathrm{m}^{2}$ regardless of glycemic control and for Asian patients with poor glycemic control and BMI of $32.5-37.4 \mathrm{~kg} / \mathrm{m}^{2}$ [6]. Even in the Standards of Medical Care in Diabetes-2020, metabolic surgery is recommended as a treatment option for T2D in Asian patients with BMI $\geq 37.5 \mathrm{~kg} / \mathrm{m}^{2}$ and in Asian patients with BMI of $32.5-37.4 \mathrm{~kg} / \mathrm{m}^{2}$ in whom non-surgical treatments have not led to sustained weight loss and improvement of complications, including hyperglycemia [31].

\section{Indications of metabolic surgery in Japan}

The indications for metabolic surgery according to the Guidelines for Safe and Outstanding Surgical Treatments for Severe Obesity in Japan (2013 edition) are patients with primary obesity aged $18-65$ years, who have severe obesity where non-surgical medical treatments given for $\geq 6$ months do not lead to significant weight loss or improvement in obesity-related health disorders [32]. Taking into consideration that obesity-related health disorders are more likely to occur in Asian people with mild BMI than in Western people, the Guidelines recommend surgery for (1) obese patients with $\mathrm{BMI} \geq 35 \mathrm{~kg} / \mathrm{m}^{2}$ if the main purpose is weight loss, and (2) obese patients with BMI $\geq 32 \mathrm{~kg} / \mathrm{m}^{2}$ that have diabetes or two or more nondiabetic obesity-related health disorders. The Guidelines for the Management of Obesity Disease 2016 set target weight loss at $\geq 3 \%$ of the current weight for obese patients and $5-10 \%$ of the current weight for severely obese patients but indicate surgical treatments for patients with $\mathrm{BMI} \geq 35 \mathrm{~kg} / \mathrm{m}^{2}$ that cannot achieve any significant weight loss or improvement of obesity-related health disorders by non-surgical medical treatments [7]. According to the Diabetes Treatment Guidelines 2019, metabolic surgery has a weight loss effect and improves diabetes, and is effective for T2D patients with severe obesity who struggle to lose weight when patient safety is ensured and an appropriate support system is in place for the patient before and after operation [9].

In Japan, LSG has been covered by government health insurance since 2014 for severe obesity patients $\left(B M I \geq 35 \mathrm{~kg} / \mathrm{m}^{2}\right.$ ) with one or more comorbidities; diabetes, hypertension, dyslipidemia, and obstructive sleep apnea syndrome (OSAS) for which non-surgical medical treatments administered for $\geq 6$ months is not sufficiently effective. In addition, the 2020 revision of health insurance has extended coverage for LSG to obese patients with a BMI of $32.5-34.9 \mathrm{~kg} / \mathrm{m}^{2}$ and $\mathrm{HbA} 1 \mathrm{c} \geq 8.4 \%$ for whom non-surgical medical treatments administered for $\geq 6$ months is not sufficiently effective. While the extended coverage has been additionally limited to patients with one or more comorbidities; (1) uncontrollable hypertension (a systolic blood pressure of $\geq 160 \mathrm{mmHg}$ despite receiving antihypertensive drugs for $\geq 6$ months), (2) uncontrollable dyslipidemia (a low-density lipoprotein [LDL]-cholesterol level of $\geq 140 \mathrm{mg} / \mathrm{dL}$ or a non-highdensity lipoprotein [HDL] cholesterol level of $\geq 170 \mathrm{mg} /$ $\mathrm{dL}$ despite receiving statins for $\geq 6$ months), or (3) severe OSAS (an apnea-hypopnea index $[\mathrm{AHI}] \geq 30$ ), no scientific evidence for this limitation has been presented. 


\section{Efficacy of metabolic surgery for T2D}

In western countries, it has been demonstrated that metabolic surgery for severe obesity can allow patients to maintain long-term weight loss and improve obesity-related health disorders $[33,34]$. It has been reported that the odds ratio for T2D remission is 9.8-15.8 compared to non-surgical medical treatments [35]. The Swedish Obese Subjects (SOS) study on diabetes up to 15 years has shown that surgical treatments significantly suppress the onset of diabetes and relieve diabetes compared to non-surgical medical treatments [3]. Moreover, the SOS study found that surgical treatments have substantially better hazard ratios than non-surgical medical treatments, for all-cause mortality at 0.77 , for cardiovascular mortality at 0.70 , and for cancer mortality at 0.77 , and that the mean adjusted life expectancy was 3 years longer with surgical treatments [36]. Other reported effects of metabolic surgery include benefits such as decreased risk of macrovascular diseases, reduced incidence rate of microvascular diseases, improvement of albuminuria and glomerular hyperfiltration, and improvement in quality of life [37-42].

The favorable efficacy of LSG for weight loss and T2D has been reported in Japan as well [43-47]. In a study involving 831 Japanese patients with severe obesity (mean BMI: $42 \mathrm{~kg} / \mathrm{m}^{2}$ ) who had undergone metabolic surgery, the results were favorable, with a percent total weight loss 3 years after operation of $33 \%, 28 \%, 26 \%$, and $19 \%$ for LRYGB, LSG, LSG with duodenojejunal bypass (LSG-DJB), and laparoscopic adjustable gastric banding (LAGB), respectively. Moreover, the clinical remission rate of T2D (HbA1c $<6.5 \%$ and no use of diabetes medicines) was 92\%, 85\%, 71\%, and $44 \%$ for LRYGB, LSG, LSG-DJB, and LAGB, respectively [44].

The J-SMART study, which examined the surgical outcomes of 332 Japanese patients with severe obesity (mean BMI: $43.7 \mathrm{~kg} / \mathrm{m}^{2}$ ) treated by LSG, reported that 2 years after surgery, the mean weight loss was $36 \mathrm{~kg}$ and the percent total weight loss was $29.9 \%$; the clinical remission rates were $75.6 \%$ for $\mathrm{T} 2 \mathrm{D}$ (HbA1c $<6.0 \%$ and no use of diabetes medicines), $59.7 \%$ for dyslipidemia and $41.8 \%$ for hypertension, and the cut-off value of the percent total weight loss for clinical remission of diabetes was $20.8 \%$ [45]. According to patients' preoperative BMI, the subgroup analysis showed that preoperative $\mathrm{HbA} 1 \mathrm{c}$ and visceral/subcutaneous fat area ratio were higher in patients with BMI of $32.0-34.9 \mathrm{~kg} /$ $\mathrm{m}^{2}$ than in patients with higher BMI levels. Although the clinical remission rate of $\mathrm{T} 2 \mathrm{D}(52.4 \%)$ and the percent total weight loss $(22 \%)$ were worse 2 years after LSG, the decrease in HbA1c level in these patients was better [48]. In addition, the reduction in the frequency of use of diabetes medicines and insulin usage was comparable or better compared to that in patients with higher BMI. A long-term coexistence of high BMI and poor glycemic control further promotes the development and exacerbation of various complications related to obesity and diabetes. When weight loss and glycemic control improvement are insufficient even after specialized non-surgical medical treatments for 6 months, metabolic surgery should be considered a treatment option without unnecessarily leaving the condition untreated.

\section{Evaluation of mental health and exclusion criteria in introduction of metabolic surgery}

2.3. Preoperative mental health assessment is essential to screen patients for the exclusion criteria from metabolic surgery and to provide them with preoperative and postoperative psychological support. (recommendation)

2.4. Mental health assessment should be performed by a mental health professional with knowledge and experience in treating obesity. (recommendation)

2.5. The patient's mental health should be comprehensively evaluated via psychological interviews and questionnaires that determine the patient's psychiatric symptoms, eating behavior, and personality. (recommendation)

2.6. All healthcare professionals involved in the assessment and treatment of obesity must treat their patients with dignity and respect to eliminate the stigma related to obesity. (recommendation)

\section{Necessity of mental health evaluation}

Obesity is a multifactorial disorder for which onset and course depend on various genetic, physiological, behavioral, psychosocial, and environmental factors, which differ between individuals [49]. There is a strong association between obesity and a number of mental disorders [50].

The prevalence of mental disorders in Japanese patients indicated for metabolic surgery has been reported to be 26-52\% [23, 48, 51]. Of these, mood disorders, including depression and bipolar disorder, are the most common, accounting for $25-30 \%$ of all cases of mental disorder, followed by anxiety disorder, intellectual disorder, and eating disorder $[48,51]$. It is relatively common for patients considering surgery to discover for the first time that they have a mental disorder [23]. It has also been reported that patients with insufficient weight loss after surgery often have personality traits such as poor spontaneity and a strong tendency toward avoidance [51]. However, it has also been revealed that behavioral and psychosocial problems such as treatment adherence, eating behavior, emotional control, and social support positively affect the postoperative clinical course [52-54]. 
In this manner, the presence of mental disorders, certain personality traits (patterns or tendencies of thoughts, emotions, and behaviors that characterize an individual), and behavioral/psychosocial problems can affect the postoperative outcome, making preoperative mental health assessment indispensable [55, 56]. This preoperative assessment allows patients to be screened for mental health characteristics that may exclude them from undergoing surgery [57]. It also allows healthcare professionals to deepen their understanding of their patients and to support patients with mental health issues by building a treatment relationship based on trust [58, 59]. In addition, sharing information about a patient's mental health with medical staff can allow the smooth implementation of various supportive measures, including psychological support aimed at the long-term maintenance of body weight after surgery $[58,59]$.

\section{Mental health evaluators and persons to evaluated}

Given the importance of preoperative mental health evaluation, the U.S. guidelines for metabolic surgery state that comprehensive behavioral and psychosocial assessments should be performed for all patients facing metabolic surgery $[55,60]$. This evaluation is best conducted by a mental health professional with training in behavioral medicine [60]. In Japan, it is recommended that mental health evaluation of patients facing metabolic surgery be performed by mental health professionals (psychosomatic physicians, psychiatrists, and licensed psychologists) who have clinical experience with the behavioral and psychosocial problems related to obesity and metabolic surgery. In principle, it would be ideal for all patients facing surgery to be evaluated by a mental health professional. Unfortunately, facilities that have no mental health professionals and others that have a shortage of such specialists have their general physicians and

Table 1 Preoperative evaluation of mental health for metabolic surgery candidates

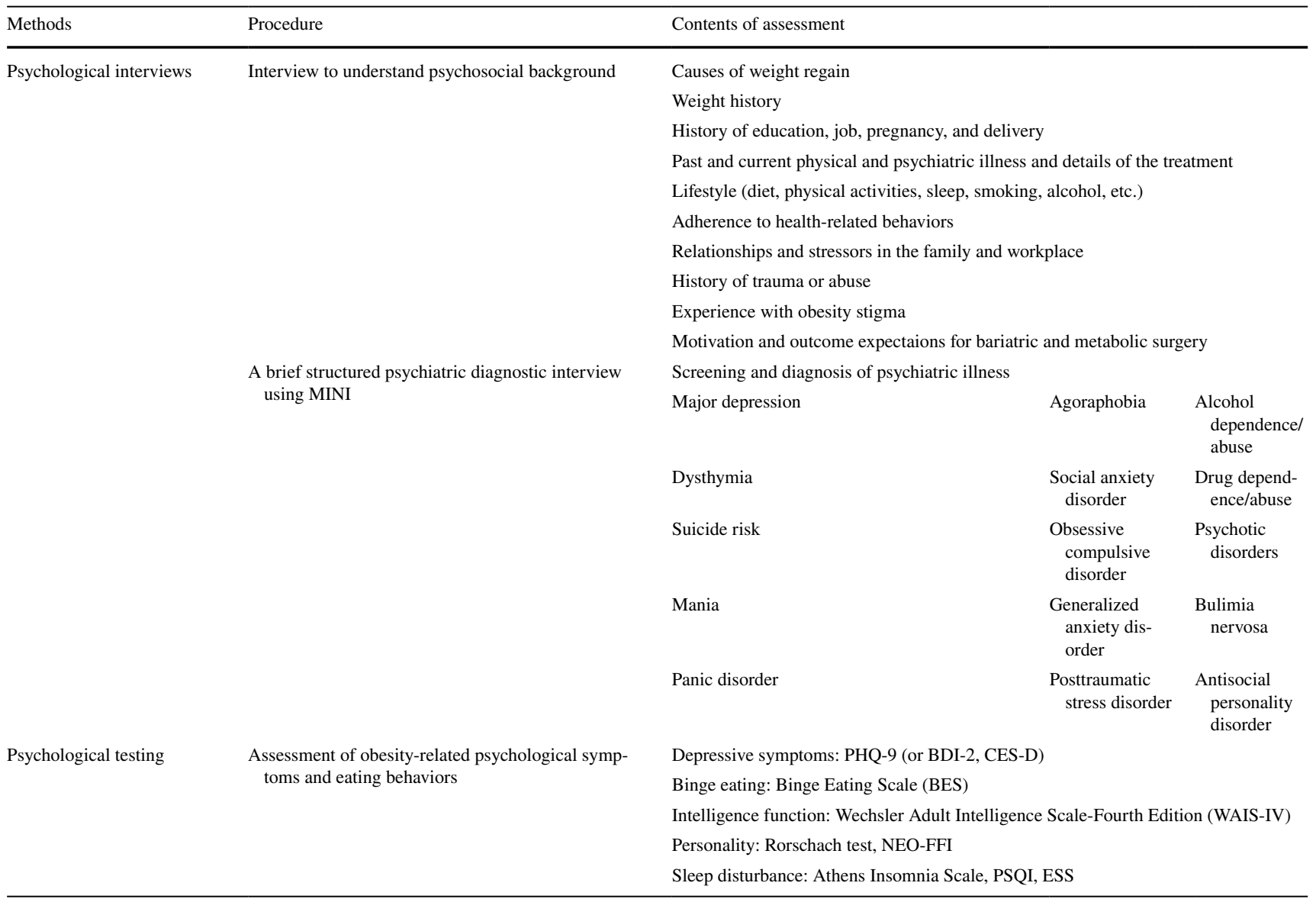

MINI Mini International Neuropsychiatric Interview, $P H Q-9$ Patient Health Questionnaire-9, BDI-2 Beck Depression Inventory-2, CES-D The Center for Epidemiologic Studies Depression Scale, NEO-FFI Neuroticism-Extraversion-Openness Five-Factor Inventory, PSQI Pittsburgh Sleep Quality Index, ESS Epworth Sleepiness Scale 
surgeons assessing patients through usage of questionnaires, patient medical history, and interviews about the patient's psychosocial background. In such cases, the examinations identify red flags, the patient is referred to an affiliated mental health professional.

\section{The evaluation of mental health}

Mental health is evaluated through psychological interviews, questionnaires on psychiatric symptoms and eating behavior, and objective tests that assess personality (Table 1) [57]. In psychological interviews, the cause of the patient's weight gain (psychosomatic correlation) is explored through an evaluation of the patient's psychosocial background. Patients are asked questions about the evolution of their body weight, their employment and education history, their history of pregnancy and childbirth, past and existing physical and mental disorders and treatments (medical history), lifestyle habits (such as meals, exercise, sleep schedules, and smoking and drinking habits), adherence to health-related behaviors, relationships and stresses within the family and in the workplace, the presence or absence of past trauma or abuse, experience with being stigmatized by their obesity (social prejudice/discrimination and self-inflicted stigma related to obesity), and their motivation and expectations related to the surgical treatment [61].

The interviewer must understand the obesity stigma and always treat patients with dignity and respect [62-65]. Patients often have idealized and unrealistic expectations that surgery will improve all aspects of their medical problems and life [66]. Rather than changing these expectations, the interviewer first needs to understand the patient's aspirations and motivation for undergoing surgery. The interviewer should provide information about the potential risks after weight loss, considering the patient's expectations. It is essential to provide adequate psychoeducation while managing the gap between expectations and weight loss outcomes [60].

Because effective weight loss through surgery only can be achieved if the patients acquire proper health behavior adherence habits, the evaluation of preoperative adherence is an essential parameter for determining a patient's suitability for surgery [60]. It should also be emphasized that surgery is not just a means of achieving weight loss, but an opportunity to review and correct previous unhealthy lifestyle habits [54].

\section{Diagnosis of mental disorders}

While structured or semi-structured clinical interviews based on DSM-5 are preferable for diagnosing mental disorders, the Mini International Neuropsychiatric Interview (MINI) is recommended for convenience [67]. The reliability and validity of MINI for Japanese patients have been established. Using MINI, most common disorders in mental health can be diagnosed in a short time (within $15 \mathrm{~min}$ ). They include major depression, risk of suicide, mania, obsessive-compulsive disorder, posttraumatic stress disorder (PTSD), alcohol dependence/abuse, drug dependence/abuse, psychotic disorder, bulimia nervosa, generalized anxiety disorder, and antisocial personality disorder. The interview also has the advantage that it does not need to be conducted by a mental health professional, which makes easy for non-specialists to screen patients.

Useful information can be obtained from self-administered questionnaires and psychological tests for the evaluation of psychiatric symptoms in general and disordered eating behavior specifically [23, 68]. It is beneficial to assess depression and binge eating, which frequently coexist with severe obesity, given that these symptoms could impact the postoperative outcome [69]. Although depression symptoms in patients undergoing weight loss and metabolic surgery are often evaluated using the Beck Depression Inventory-2 (BDI-2) and the Center for Epidemiologic Studies Depression Scale (CES-D), we recommend the Patient Health Questionnaire-9 (PHQ-9) because of its simplicity [70]. It also has the advantage of complying with the American Psychiatric Association's mental disease classification and diagnostic criteria. A score $\geq 10$ indicates the presence of depression symptoms, and a score $\geq 15$ indicates severe depression symptoms. Depression is often associated with sleep disorders, and patients with severe obesity frequently co-develop OSAS; hence, it is beneficial to evaluate sleep rhythms using scales such as the Athens insomnia scale, the Pittsburgh Sleep Quality Index, or the Epworth Sleepiness Scale [71].

The Binge Eating Scale (BES) is recommended for the evaluation of binge eating [72]. A score $\geq 18$ indicates moderate binge eating, while a score $\geq 27$ indicates severe binge eating. Furthermore, evaluating a patient's intellectual capacity (WAIS-IV) and assessing the patient's personality using tests, such as the Rorschach test and the NEO-FFI Personality Inventory, provide critical information about the need for psychological intervention before and after surgery to support a positive outcome of the surgical treatment [73-75].

\section{Exclusion criteria for metabolic surgery from a mental health perspective}

2.7. The exclusion criteria for metabolic surgery should be judged individually according to the degree and condition of the psychiatric symptoms, rather than making decisions in a disease-specific manner. (recommendation)

2.8. The main exclusion criteria for surgery should consider current or recent drug and/or alcohol depend- 
ence/abuse and psychiatric disorders that are untreated or not stable even after treatment. (consideration)

2.9. Criteria that would lead to consideration of surgery deferral or discontinuation include, among several others, suicidal thoughts, severe mental retardation, and lack of understanding of the risks of surgery. (consideration)

\section{Relation between postoperative outcome and mental disorders}

While multicenter and single-center studies in Japan have identified high rates of comorbidity of mental disorders in patients with insufficient weight loss or excessive weight loss after metabolic surgery, there was no difference in the extent of weight loss according to the presence or absence of mental disorders identified before surgery [ 45 , 48]. While some reports indicate that mental disorders are a risk factor in the postoperative outcome, there is ample evidence suggesting that surgery leads to weight loss and improved mental health and QOL for patients with mental disorders [76-79]. Therefore, the exclusion criteria for metabolic surgery in relation to mental health status should be carefully evaluated and determined for each individual according to the extent of the patient's psychiatric symptoms and environment, rather than in a disease-specific manner [77]. In the current European and American guidelines, no consensus has been established on the exclusion criteria related to the mental health of metabolic surgery patients [60, 76-79].

\section{Exclusion criteria for metabolic surgery}

The main exclusion criteria for metabolic surgery are (1) current or recent drug or alcohol dependence/abuse and (2) a mental disorder that is untreated or not stable even after treatment (such as depression, bipolar disorder, schizophrenia, or bulimia nervosa) $[54,55,77,79]$. The main drugs that cause dependence and addiction are narcotics, stimulants, cannabis, opioids, and benzodiazepines. Past drug or alcohol dependence/abuse does not necessarily affect the postoperative risk of recurrence or weight loss and are, therefore, not part of the exclusion criteria for surgery [80]. However, the patients are required to discontinue drug and alcohol use for at least 1 year before surgery $[80,81]$. If a patient is currently undergoing treatment for a mental disorder, it is best to contact the patient's attending psychiatrist to request information about their treatment status [82]. The patient will not violate the exclusion criteria if they are receiving proper treatment and if their symptoms are stable.

\section{Situations that require careful consideration of postponement or discontinuation of metabolic surgery}

Situations that warrant possibly deferring or rescinding metabolic surgery include (1) a history of multiple suicide attempts or recent suicidal thoughts/intention, (2) reluctant attitude/poor adherence to the tasks recommended before and after surgery (such as dietary management, exercise, and abstinence from smoking and drinking alcohol), (3) severe mental retardation (IQ < 50), (4) borderline personality disorder, 5) lack of understanding about the risks and benefits of surgery, (6) lack of willingness to participate in longterm follow-up, (7) serious stress levels in daily life, and (8) inability to manage self-care, and lack of a family member/supporter who can assist with long-term care (Table 2) $[60,76,78,79]$. While binge-eating disorder and nocturnal eating syndrome do not prevent patients from undergoing surgery, their preoperative evaluation is essential, because

Table 2 Checklist for the clinical judgment of contraindications to metabolic surgery from the viewpoint of mental health

\begin{tabular}{ll}
\hline Item & Checklist \\
\hline Conraindications to surgery & $\begin{array}{l}\text { Current or recent drug and/or alcohol dependence/abuse } \\
\text { Untreated or unstable psychiatric disorders (depression, bipolar disorders, schizophrenia, } \\
\text { bulimia nervosa, etc.) and psychosis, untreated bulimia nervosa, and intractable sub- } \\
\text { stance or alcohol abuse } \\
\text { History of multiple suicide attmpts or a recent suicide ideation/intent } \\
\text { Factors that can postpone or not allow surgery }\end{array}$ \\
$\begin{array}{l}\text { A reluctance to adhere to the postoperative recommendations } \\
\text { Severe mental retardation (IQ }<50)\end{array}$ \\
$\begin{array}{l}\text { Borderline pesonality disorder } \\
\text { A lack of understanding regarding the risks, benefits, and results of the surgical procedure } \\
\text { A lack of willingness to participate in prolonged medical follow-up } \\
\text { Current severe life stressors } \\
\text { A lack of patient self-care or long-term family or social support that will warrant such care }\end{array}$
\end{tabular}


they affect long-term postoperative weight management and psychiatric symptoms [77, 79].

The items mentioned above do not necessarily dictate exclusion or deferral of surgery: the final decision should be based on the advice of a mental health professional with experience in treatment of obesity. The patient must be prepared to receive psychological support before and after surgery, and the medical system must adequately and appropriately respond to the patient's mental health status [83].

\section{Requirements for the introduction of metabolic surgery}

2.10. It is recommended that institutions performing metabolic surgery have a multidisciplinary medical team of specialists with knowledge and experience in providing nursing, nutritional and exercise guidance, and mental and psychological support. (recommendation)

2.11. It is recommended that metabolic surgery is performed by a surgeon with sufficient knowledge and experience in the procedures. (recommendation)

\section{Requirement for the institutions}

To achieve the aims of metabolic surgery, it is important to ensure perioperative safety and establish a long-term follow-up system. Therefore, it is indispensable to ensure the collaboration of physicians, psychiatrists, surgeons, and other medical professionals with a team core of diabetologists certified by the JDS and obesity specialists certified by the JASSO [8, 9]. Diabetologists and obesity specialists should identify patients from their routine diabetes and obesity medical practices for whom metabolic surgery would be an adequate treatment option. They should then determine the suitability of metabolic surgery for each patient through close consultation and cooperation with the surgeons who will perform the surgery, and formulate and carry out the pre- and post-operative medical treatment plans.

The institution should meet the requirements for a Japanese center of excellence certificated by the JSTO. In particular, there should be (1) a medical team in place that can comprehensively provide special nursing, nutritional and exercise guidance, and mental/psychological support; (2) equipment/facilities in place that are needed for safe management of the obese patients; (3) a system in place for holding regularly preoperative evaluation meetings and meetings about accidents during operation and postoperative complications and considering the patient's opinion in making the treatment decision; (4) a system in place for longterm follow-up of the patient undergoing surgery; and (5) a patient support group in place [32, 84-89]. It is desirable for the team members to attend regularly educational programs such as the comprehensive treatment seminars for obesity certificated by the JSTO and for the institution to get a Japanese center of excellence certification by the JSTO when a multidisciplinary medical team provides the patient nursing, nutritional and exercise guidance, and mental and psychological support for metabolic surgery [88, 89].

It is desirable to carry out the postoperative follow-up in each medical institution under the supervision of diabetologists and obesity specialists by a multidisciplinary medical team including certified Japanese diabetes educators (Certified Diabetes Educators of Japan, established jointly by the three academic societies: the JDS, the Japan Society of Metabolism and Clinical Nutrition, and the Japan Academy of Diabetes Education and Nursing). Such educators should have attended educational programs on metabolic surgery conducted by the JSTO or should have regularly gone through the e-learning training programs on metabolic surgery conducted by the JASSO and the JDS $[8,9]$.

\section{Requirements for surgeons}

The requirements for surgeons include the following: (1) board-certificated surgeons by the Japan Surgical Society or the Japanese Society of Gastroenterological Surgery; (2) experience as a primary surgeon under a supervision of experienced surgeon with expertise in $\geq 20$ cases of metabolic surgery before handling the initial cases; (3) membership in the JSTO and the IFSO, and sufficient knowledge and skills related to the pathology of morbid obesity, perioperative management, surgical techniques, complications and revision surgery; (4) ideally, experience in observation and training in a Japanese center of excellence certificated by the JSTO before introducing the metabolic surgery; and (5) completion of educational seminars and training courses related to metabolic surgery recommended by societies such as the JSTO, the JDS, the JASSO, the Japan Society for Endoscopic Surgery, or the IFSO [32, 87-89].

\section{Surgical procedure for metabolic surgery}

2.12. The main surgical procedures of metabolic surgery used in Japan are LAGB, LSG, LRYGB, and LSGDJB.

The objective of metabolic surgery is to achieve weight loss based on restriction of food intake by making the smaller stomach size, suppression of food digestion and absorption by bypassing the gastrointestinal tract (small intestine) or the combination of the two methods. Restrictive procedures include LAGB and LSG, while the combination procedures of restriction and malabsorption are LRYGB and LSG-DJB (Fig. 2) [32, 90, 91]. In Japan, most procedures 
adjustable gastric banding

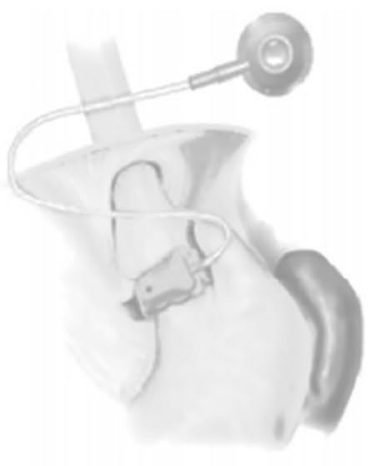

sleeve gastrectomy

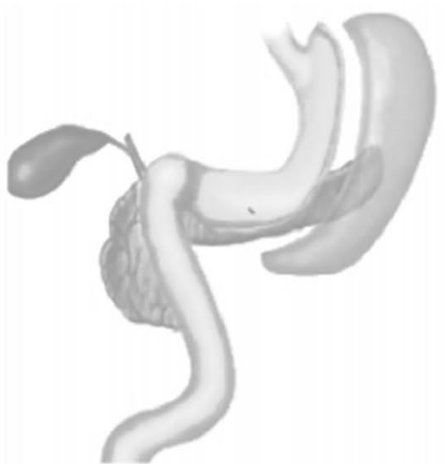

Roux-en-Y gastric bypass

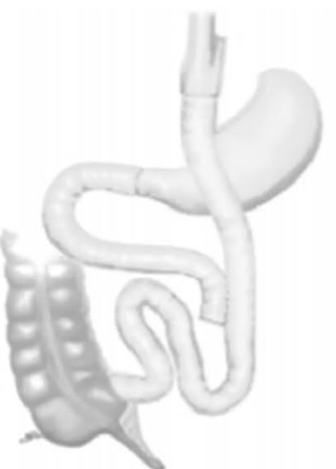

sleeve gastrectomy with duodenojejunal bypass

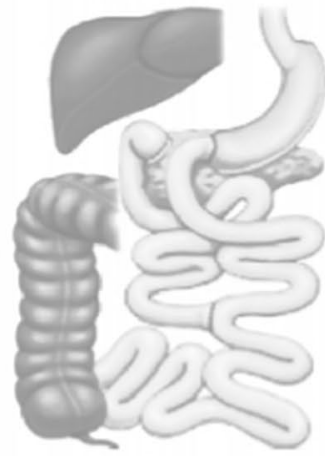

Fig. 2 Surgical procedures in Japan

Table 3 Features of major surgical procedures in Japan

\begin{tabular}{|c|c|c|c|}
\hline Surgical procedures & $\begin{array}{l}\text { Percentage of excess weight } \\
\text { loss } 3 / 5 \text { years after surgery }\end{array}$ & Advantages & Complications cautions \\
\hline Sleeve gastrectomy & $68 / 66$ & $\begin{array}{l}\text { Metabolic effects } \\
\text { High safety due to no anastomosis } \\
\text { Fewer early reoperations }\end{array}$ & $\begin{array}{l}\text { Reflux esophagitis } \\
\text { Gastric tube stenosis } \\
\text { Refractory anastomotic leakage }\end{array}$ \\
\hline Roux-en-Y gastric bypass & $81 / 78$ & $\begin{array}{l}\text { Metabolic effects are higher than LSG } \\
\text { Effective for GERD }\end{array}$ & $\begin{array}{l}\text { Suture failure is about } 4 \% \\
\text { Anastomotic stenosis and ulcer } \\
\text { Internal hernia } \\
\text { Small bowel obstruction } \\
\text { Micronutrient deficiency }\end{array}$ \\
\hline $\begin{array}{r}\text { Sleeve gastrectomy with } \\
\text { duodenojejunal bypass }\end{array}$ & $86 / 80$ & $\begin{array}{l}\text { Metabolic effects are higher than LSG } \\
\text { Preservation of pyloric ring and gastric motility } \\
\text { Avoidance of dumping symptoms }\end{array}$ & $\begin{array}{l}\text { Reflux esophagitis } \\
\text { Gastric tube stenosis } \\
\text { Limited number of facilities } \\
\text { Surgical difficulty is high }\end{array}$ \\
\hline
\end{tabular}

of metabolic surgery are laparoscopically performed, and in 2020, LSG accounted for $94.9 \%$, LSG-DJB $2.3 \%$, and LRYGB $0.2 \%$, respectively [90]. The features of the main surgical procedures are shown in Table 3.

\section{(a) Adjustable gastric banding}

A silicone band is wrapped around the upper part of stomach to create a stomach pouch of around $30 \mathrm{~mL}$. The balloon inside the band is inflated by injecting physiological saline from a reservoir to adjust the band's tightness around the stomach.

\section{(b) Sleeve gastrectomy}

The greater curvature of the stomach is removed to leave only the banana-shaped lesser curvature (about $100 \mathrm{~mL}$ ), and this is a safe operation without gastrointestinal anastomosis. LSG was developed as the initial bariatric surgery to avoid complications in biliopancreatic diversion with duodenal switch for super obese patients. It is currently the most frequently performed procedure due to its good weight loss and improvement in obesity-related health disorders $[44,90]$.

(c) Roux-en-Y gastric bypass

A stomach pouch of around $50 \mathrm{~mL}$ is created in the upper part of stomach, and gastrojejunostomy and jejunojejunostomy are performed. In addition to restrictive food intake, absorption is suppressed by the inability of food passage from the remnant stomach and duodenum to the biliopancreatic tract. However, because an excluded distal remnant stomach will remain, it is not considered a suitable surgical procedure in Japan, where there are many gastric cancer cases. This technique is chosen in patients with preoperative reflux esophagitis or in patients with severe diabetes in whom LSG cannot achieve clinical improvement. 
(d) Sleeve gastrectomy with duodenojejunal bypass

This is a modified method of biliopancreatic diversion, in which jejunal bypass is performed in addition to LSG [92]. This procedure was developed in Japan, where gastric cancer is common. It is a surgical procedure that provides malabsorption in addition to intake restriction, and it is expected to offer a weight-loss effect and clinical remission of diabetes similar to LRYGB. It is performed as advanced medical treatment in patients with severe diabetes who are not expected to achieve clinical remission of diabetes by LSG [46].

\section{Prediction of the metabolic surgery effects and selection of the surgical procedure}

\section{Factors affecting T2D improvement after metabolic surgery}

\section{Patients who are expected clinical remission after metabolic surgery}

3.1. Patients with a relatively retained insulin secretory capacity, and with a high insulin resistance associated with obesity, not patients resistant to medical treatment, are expected clinical remission; hence, metabolic surgery is recommended. (recommendation)

\section{Patients who are expected dose reduction of accustomed medication even without achievement of clinical remission}

3.2. Patients with long history of diabetes and with reduced insulin secretory capacity could benefit from metabolic surgery with regard to the marked reduction of administered insulin doses or other medications for diabetes, even if no clinical remission is achieved. Therefore, it is advisable to consider the metabolic surgery for these patients. (consideration)

Metabolic surgery is known to be highly effective for T2D improvement [33, 39, 93-95]. So-called clinical remission, in which glycemic control is normalized (no use of diabetes medication, and $\mathrm{HbA} 1 \mathrm{c}<6.5 \%$ ), can be achieved in many patients after metabolic surgery [33, 39, 93-95].

However, there are some cases in which the improvement effect is insufficient after surgery, or there are some cases relapsing diabetes in the long term. Therefore, predicting the effect of metabolic surgery on diabetes before surgery is useful for selecting the surgical procedure or determining the timing of surgery. Even if a patient does not achieve clinical remission after surgery, the medication dosages could be reduced, and glycemic control could be improved; thus, surgery would be considered beneficial from both medical economics and medical complication risk reduction perspectives.

Many studies have reported the clinical factors predicting the improvement of diabetes after metabolic surgery. The most representative predictive factors of efficacy include age, preoperative body weight/BMI, sex, serum C-peptide concentration, disease period of diabetes, preoperative HbA1c level, history of insulin use, type/doses of diabetes medications used, preoperative fasting blood glucose level, and surgical procedure [96-103].

Among them, a high probability of diabetes remission is expected in the cases with the following qualities which reflect the insulin secretory reserve: patients with highserum C-peptide concentrations; those with a short duration of diabetic disease; young ages; patients with no history of insulin use; and patients who are expected the improvement of insulin resistance after surgery [96-102].

In addition, although improvement is more substantial in patients with high BMIs reflecting preoperative high insulin resistance, metabolic surgery has also proven to be highly effective for patients with relatively low body weights (BMIs of approximately $30 \mathrm{~kg} / \mathrm{m}^{2}$ ) in recent years [97, 98, 104, 105]. Hence, the body weight and BMI are not considered to be critically important predictive factors of efficacy of metabolic surgery.

These results suggest that metabolic surgery will be highly effective in patients with a relatively retained insulin secretory capacity and with a high insulin resistance which is expected to be improved by reducing the body weight, rather than in cases in which glycemic control has been poor for a long time and non-surgical treatments become ineffective, or in those requiring insulin administration because of their impaired insulin secretory capacity.

However, it is also important to create a consensus on medical and economic usefulness for cases with a long history of diabetes and decreased insulin secretory capacity, even if the diabetic remission is not achieved, since an insulin dosage or a number of medications could be reduced.

\section{Scoring system predicting the T2D improvement after metabolic surgery}

\section{Representative predictive scores}

3.3. Various predictive scores consisting of several factors have been proposed to predict clinical improvement of diabetes after surgery. Representative scores include the ABCD score, the DiaRem score, the Advanced DiaRem score, the IMS score, and the DiaBetter score. Each of these scores effectively predicts clinical improvement of diabetes 


\section{Predictive scores suitable for Japanese patients}

3.4. The ABCD score is highly objective for each factor, is easy to calculate, and has excellent predictability for clinical remission. It was created based on data from Asian patients, including Japanese, making it a suitable predictive score for Japanese patients. (recommendation)

A single predictive factor alone does not highly predict the clinical improvements that might occur postoperatively. The prediction accuracy increases through comprehensive judgments with combined several elements. Thus, several predictive scores that combine multiple factors have been proposed.

Major predictive scores include the ABCD score (age, BMI, serum C-peptide concentration, and the duration of diabetes) [103, 106-108], the DiaRem score (age, preoperative $\mathrm{HbA1c}$ level, the history of insulin use, and types of diabetes medications used) [109], the Advanced DiaRem score (age, preoperative HbA1c level, history of insulin use, presence or absence of oral diabetes medicines used and their numbers, and the duration of diabetes) [110], the IMS score (preoperative $\mathrm{HbAlc}$ level, the duration of diabetes, a number of diabetes medicines other than insulin, and the history of insulin use) [111], and the DiaBetter score (preoperative $\mathrm{HbA1c}$ level, the duration of diabetes, types of diabetes medications used) [112], and each of them can effectively predict clinical improvements after surgery (Table 4).

These scoring systems do not simply predict the efficacy of surgery, but also serve as indicators for selecting a surgical procedure (e.g., when using the IMS score, the surgical procedure is recommended according to the number of points) [111]. LSG-DJB, which has been approved as an advanced medical treatment in Japan since 2018, suggest to use the $\mathrm{ABCD}$ score and the history of insulin use as indicators for appropriate selection [46].

The ABCD score is based on data from multiple institutions in Asia, whereas the DiaRem score, the Advanced DiaRem score, and the IMS score were created based on case data from the United States and Europe. It is important that, in addition to their predictability, the score and remission rate correlate well, the score can be calculated based on objective indicators, and it can be calculated easily.

Factors such as the type of diabetes medication used in the DiaRem score may be less objective, because the choice reflects the treating physician's policy. Also, the IMS score is reported not to correlate well with clinical improvement of diabetes in Asian patients [47]. On the other hand, there is a report of multicenter study in East Asia indicating that the IMS score was as useful as the ABCD score [113].

The ABCD score is highly objective for each factor, is easy to calculate, and has excellent predictability for clinical remission of diabetes. It has been created based on data of Asian patients, including Japanese, which makes it a predictive score suitable for Japanese patients [44, 46, 47, 112-117].

\section{Selection of surgical procedure according to the diabetes remission predictive score}

\section{Diabetes remission predictive score suitable for selecting the surgical procedure for Japanese patients}

3.5. The $\mathrm{ABCD}$ score is recommended as a diabetes remission predictive score to use when selecting the surgical procedure. (recommendation)

\section{Patients indicated for SG}

3.6. SG is recommended for obese patients with $\mathrm{T} 2 \mathrm{D}$ with a short duration of diabetes and a well-retained insulin secretory capacity. (recommendation)
Table 4 Predict scoring system of T2D resolution after surgery and its predictor

\begin{tabular}{|c|c|c|c|c|c|c|c|c|}
\hline & Age & $\begin{array}{l}\text { Preop- } \\
\text { erative } \\
\text { BMI }\end{array}$ & $\begin{array}{l}\text { Preop- } \\
\text { erative } \\
\text { C-peptide }\end{array}$ & $\begin{array}{l}\begin{array}{l}\text { Dura- } \\
\text { tion of }\end{array} \\
\text { DM }\end{array}$ & $\begin{array}{l}\text { Number of } \\
\text { DM medi- } \\
\text { cine }\end{array}$ & $\begin{array}{l}\text { Type of } \\
\text { DM medicine }\end{array}$ & Insulin use & $\begin{array}{l}\text { Preop- } \\
\text { erative } \\
\text { HbA1c }\end{array}$ \\
\hline $\mathrm{ABCD}$ & O & 0 & 0 & 0 & & & & \\
\hline DiaRem & O & & & & & 0 & O & 0 \\
\hline Ad-DiaRem & O & & & 0 & O & O & 0 & 0 \\
\hline IMS & & & & 0 & 0 & 0 & 0 & 0 \\
\hline DiaBetter & & & & 0 & & 0 & & 0 \\
\hline
\end{tabular}




\section{Gastrointestinal bypass surgery suitable for Japanese patients, and patients for whom gastrointestinal bypass surgery should be considered}

3.7. Because the remission rate of diabetes is higher in the cases with a surgery that add the malabsorptive procedure such as gastrointestinal bypass, it is advisable to consider carrying out the gastrointestinal bypass surgery for patients with reduced insulin secretory capacity. (consideration)

3.8. When selecting the gastrointestinal bypass surgery, consider LSG-DJB as the first choice, because it is currently an only approved procedure as an advanced medical treatment in Japan. (consideration)

3.9. Consider selecting LSG-DJB for obese T2D patients with an $\mathrm{ABCD}$ score of $<5$ points or with insulin use. (consideration)

The ABCD score (maximum of 10 points), calculated using age, BMI, serum C-peptide concentration, and duration of diabetes is simple and highly objective. It correlates well with the postoperative outcome for Japanese patients, and is widely used in clinical practices [44, 46, 47, 113].

The main surgical procedures currently being performed can be classified as restrictive procedures which limits food intake, and surgeries adding the malabsorptive procedures (e.g., the gastrointestinal bypass surgery) [32]. In Japan, as of 2020, LSG is covered by the National Health Insurance, and LSG-DJB is approved as the advanced medical treatment; metabolic surgery is otherwise privately funded. Since it is difficult to observe endoscopically the distal excluded remnant stomach after LRYGB, which is the most popular malabsorptive procedure in western countries, only a limited number of institutions in Japan have introduced this surgery because of concerns about delays in discovering gastric cancer. In contrast, LSG-DJB is considered as a bypass surgery suitable for Japanese patients, because of the simplicity of endoscopic observation of the remnant stomach, besides high efficacy on diabetes remission and body weight loss $[92,118]$.

LSG is the most commonly practiced surgical procedure globally, and it is covered by National Health Insurance in Japan. The clinical improvements observed in 183 Japanese patients with obesity and T2D at 3 years after LSG correlated well with their ABCD scores [44]. The outcomes, including the weight loss effect, were similar to those seen in overseas reports. Furthermore, the J-SMART study, which analyzed the postoperative clinical outcomes of 322 Japanese patients with severe obesity (mean BMI of $43.7 \mathrm{~kg}$ / $\mathrm{m}^{2}$ ) who underwent LSG at 10 institutions certified by the JSTO, showed that the percentage of total weight loss correlated with the clinical improvement of diabetes and that the cutoff value of the percentage of total weight loss for clinical remission was $20.8 \%$ [45]. Furthermore, in a multicenter study involving 298 patients who underwent LSG or LSG-DJB at 8 institutions in Japan, the clinical remission rate of diabetes at 1 year after LSG was $95.0 \%$ in patients who had $A B C D$ scores $\geq 6$ points and relatively retained insulin secretory capacity, which is almost equivalent to SG-DJB.

However, LSG-DJB leads to a higher level of clinical improvement than the SG alone for patients with impaired insulin secretory capacity, such as patients with an ABCD score $\leq 5$ points or those using insulin $[46,92]$. Furthermore, in a single-center, retrospective study in Taiwan involving 579 patients, comparing the remission rates between LSG and gastric bypass at 1 year after the procedures, the remission rate of gastric bypass was higher than those of LSG in patients with low ABCD scores [107]. In general, the remission rate for $\mathrm{T} 2 \mathrm{D}$ is higher with gastrointestinal bypass surgery than with the restrictive procedure [119].

However, many of these reports are based on the short-term results of metabolic surgery. A clear conclusion about the difference in efficacy between the surgical procedures with regard to long-term clinical improvement of diabetes has not been reached yet [120, 121].

Ultimately, it is important to choose the surgical procedure according to the patient's condition, having sufficiently understood each surgical procedure's characteristics, related complications, and revision surgery.

\section{Perioperative management and follow-up system}

Metabolic surgery has various unique characteristics throughout preoperative preparations and postoperative follow-up that are quite different from routine surgical procedures. Abdominal surgery on patients with severe obesity is complicated, and preoperative weight loss and nutritional assessment are recommended to reduce surgical risks. In addition, great care should be taken in preventing perioperative complications. Furthermore, since patients with morbid obesity tends to have multiple obesity-related health disorders, they need non-surgical medical care to manage the disease before surgery and long-term postoperative follow-up. Most of patients with severe obesity have mental and psychosocial problems, requiring mental health support before and after surgery. It is recommended that healthcare providers such as surgeons, physicians, mental health specialists (psychiatrists, psychosomatic physicians, and licensed psychologists), anesthesiologists, registered dietitians, nurses, physiotherapists and social workers form a multidisciplinary team and provide the preoperative, perioperative care and postoperative follow-up to ensure the safety and effectiveness of the surgery. 
The preoperative management, described in this section, focuses on issues that should be added on the premise that "medical treatment for obese T2D" has been already performed. However, a patient, who is determined to be an indication for surgical treatment, has possibly been deferred or discontinued for surgery. The preoperative management might start at the timing of decision for undergoing surgery (generally scheduled 3 months before surgery) and up to the surgical intervention. There is no endpoint to postoperative follow-up. Instead, it is ideal for each patient to maintain a connection with healthcare providers for as long as possible, depending on situation.

\section{Preoperative management}

\section{Nutritional assessment and intervention}

4.1. Patients, who is considered for metabolic surgery, are recommended to evaluate their nutritional status through investigation of meal contents and eating habits before surgery, in addition to laboratory examinations. (recommendation)

4.2. Nutritional intervention for assessing patients' nutritional status is recommended to correct the status and prevent surgery-related complications as well as getting accustomed to have proper eating habits after surgery. (recommendation)

4.3. It is recommended that patients undergo weight loss by ingesting calorie-restricting diets just before surgery (approximately one month) to reduce the visceral fat and liver volume. (recommendation)

Since patients with obesity often have a potential malnutrition, their nutritional status would be evaluated before metabolic surgery through their meal contents and eating habits, in addition to laboratory examinations [122-125]. It has been reported that the degree of obesity is inversely correlated with levels of serum albumin and micronutrients. This is due to the peculiar eating behavior of patients with obesity, which involves consuming large amounts of processed food, few fresh fruits and vegetables, large quantities of confectionery, soft drinks containing sucrose and alcohol, and excessive eating during the night. A large food intake at one time may reduce the efficiency of protein and micronutrients absorptions, leading to a deficiency of micronutrients. The American Society for Metabolic and Bariatric Surgery (ASMBS) recommends evaluating the level of vitamin $B_{1}$, vitamin $B_{12}$, vitamin $D$, calcium, vitamin $A$, vitamin $E$, vita$\min \mathrm{K}$, folic acid, and iron levels before surgery and evaluating zinc and copper levels in patients before gastrointestinal bypass surgery [122]. Since nutritional deficiency may cause postoperative complications, it is required to correct the nutritional status before operation.

Preoperative nutritional intervention is required for achieving proper eating habits, carrying on after operation. Dietary contents change significantly after operation, leading to increase stress and confusion for the patients as well as their family. Therefore, an appropriate preoperative explanation about the postoperative variable changes, such as reduced meal intake, frequent nausea and vomiting, and changes in food preferences, as well as the possibility of nutritional disorders, small weight reduction and weight rebound is recommended in order to ascertain whether the patient will be able to tolerate the eating habits after surgery.

For patients with obesity, the working space in the abdominal cavity during laparoscopic surgery can be small because of the excessive visceral fat accumulation and enlargement of the liver due to non-alcoholic fatty liver disease (NAFLD). This often makes surgical manipulations difficult. Therefore, it is recommended that the patients lose some weight before surgery to facilitate the surgical procedures [126, 127]. It is necessary for patients to lose as much weight as possible before surgery through a calorie-limiting diet, preferably aiming for a $5 \%$ weight loss. However, it is presumed that most patients with obesity indicated for metabolic surgery will find it difficult to lose $5 \%$ of body weight even within six or more of non-surgical medical treatments before surgery. For patients who do not achieve adequate weight loss before surgery, a short period (2-4 weeks) of a low-calorie diet (LCD; less than target body weight $[\mathrm{kg}] \times 20-25[\mathrm{kcal} / \mathrm{kg}] /$ day) or very low-calorie diet (VLCD; $<600 \mathrm{kcal} /$ day) can be considered immediately (approximately one month) before surgery. A VLCD is administered in the hospital, and it is possible to maintain the required amount of protein (about $20 \mathrm{~g} / \mathrm{bag}$ ) and micronutrients by composing the diet around the formula (about $180 \mathrm{kcal} / \mathrm{bag}$ ). It has been reported that 9 weeks on an LCD (800 kcal/day) reduces BMI by $12.3 \%$ and visceral fat area by $9.2 \%$ [128]. Another report states that a calorie-limiting diet of 456-800 kcal/day for 2 weeks or more reduces liver volume by $1.6-3.4 \%$ per week [129-132]. While some reports indicate that weight loss immediately before surgery is effective for weight loss after surgery [133], some data suggest no significant correlation between weight loss before surgery and weight loss and the incidence rate of complications after surgery $[126,134]$.

On the basis of the above, the involved healthcare providers need to have a thorough understanding of metabolic surgery in performing a nutritional assessment, diagnosis, intervention, and monitoring tailored to each patient. This will secure optimal preoperative preparation to achieve the maximum weight loss effect while preventing nutritional disorders. 
(a) Type 2 diabetes (T2D)

4.4. Glycemic control is recommended to be maintained well before metabolic surgery. (recommendation)

4.5. Diabetes treatment is recommended to switch to insulin-based therapy before metabolic surgery. (recommendation)

With facing metabolic surgery, diabetic patients are often motivated to improve their glycemic control, resulting in optimizing their lifestyle and diet, including replacement with formula foods. These behavior changes might elicit improvement of glycemic control, requiring modification of diabetic treatment to prevent hypoglycemia.

According to the US guidelines, the preoperative recommended target of HbA1c is $6.5-7.0 \%$ and that of blood glucose level is $80-180 \mathrm{mg} / \mathrm{dL}$. Furthermore, preoperative $\mathrm{HbA} 1 \mathrm{c}$ level of $7.0-8.0 \%$ is recommended for patients with advanced vascular complications or suffering diabetes for long periods [127]. In the case with $\mathrm{HbAlc}>8.0 \%$, performing metabolic surgery should be carefully considered into cancel or postpone. On the other hand, preoperative glycemic targets proposed by the JDS are urinary ketone bodies-negative, fasting blood glucose of $100-140 \mathrm{mg} / \mathrm{dL}$, or postprandial blood glucose of $\leq 160-200 \mathrm{mg} / \mathrm{dL}$ [135]. Thus, optimum glycemic control for surgery should be determined based on the blood glucose level rather than that of HbA1c. If proper glycemic control cannot be achieved, inpatient treatments should be considered prior to surgery.

Before gastrointestinal surgery, most of the diabetes medications are preferable to stop [136] and make a shift to insulin treatment [135]. According to the National Health Service Guidelines from the United Kingdom, the preoperative washout period for metformin should start on the day of operation [137]. However, Japanese guidelines indicate that the washout period should start 2 days before surgery [135]. For sodium-glucose cotransporter-2 (SGLT2) inhibitors, it is recommended that the washout period begins 3 days before surgery [138].

(b) Obstructive sleep apnea syndrome (OSAS)

4.6. As OSAS is a frequent complication in patients with severe obesity, all patients are recommended to be screened and be established its diagnosis and grade as much as possible before surgery. (recommendation)

4.7. As untreated OSAS involves a high risk of perioperative complications, it is recommended that continuous positive airway pressure (CPAP) therapy should be initiated before surgery. (recommendation)

The prevalence of OSAS is reported to be $38-88 \%$ of the patients with severe obesity [139] and $>70 \%$ of patients facing metabolic surgery $[140,141]$. Patients with severe obesity have an increased prevalence of surgical complications, such as thromboembolism, infections, and postoperative mortality, and OSAS further increase their risk [142]. Therefore, to diagnose OSAS before surgery is very important. Wherever possible, all patients facing metabolic surgery are preferable to be examined a simplified respiratory polygraphy test and evaluated the possibility of having OSAS using the respiratory disability index. Moreover, screening using questionnaires is expected to increase the diagnostic rate of OSAS, and it is useful for determining the necessity of an overnight polysomnography (PSG) examination. When the results of simplified respiratory polygraphy test suggest OSAS, a patient who meet the criteria as severe grade is indicated for starting CPAP therapy, or another one, who do not meet the criteria, is preferable to undergo a PSG for both diagnosis and evaluation of severity of OSAS.

When the results of PSG confirm the diagnosis of severe OSAS, meeting the requirements for health insurance coverage of the CPAP procedure, the CPAP therapy should be started promptly. Preoperative and perioperative CPAP use can reportedly reduce hypercapnia, hypoxemia, pulmonary artery vasospasm and related cardiopulmonary complications, demonstrating its practical significance [142, 143]. The routine usage of CPAP device is expected to be brought on admission to hospital, and used before and after surgery to achieve maximum tolerance to the CPAP treatment. However, as CPAP use generates aerosol, it is necessary to keep infection control measures in mind and preferably perform the treatments in a private room [144].

(c) Handling and managing mental disorders

4.8. If the patient is receiving treatment for a mental disorder, it is recommended that the medical team reconfirm that the treatment is appropriate and that the patients' symptoms are stable before surgery. (recommendation)

4.9. If a mental disorder is identified for the first time during pre-surgery assessment, the recommendation is to put the surgery on hold and to prioritize treatment of the mental disorder. (recommendation)

As severe obesity is frequently associated with mental disorders, patients should undergo a mental health assessment before surgery. If a mental disorder is identified, it must be managed accordingly. If the patient is already receiving treatments for a mental disorder, it should be confirmed directly with an attending mental health clinician who treats mental health issues, such as psychiatry or psychosomatic medicine, whether the patients' condition is stable enough to undergo metabolic surgery. There can be gaps in awareness about mental disorders between patients and their attending clinician, because patients with mental illnesses may underplay the state of their mental disorder due to their desire to undergo surgery or their lack of ability to communicate $[54,145]$. 
If a mental disorder is discovered for the first time during the preoperative mental health assessment, its treatment should be prioritized. Many existing guidelines include the presence of a severe, untreated mental disorder in their exclusion criterion for metabolic surgery $[60,78,146]$. However, metabolic surgery should be considered, even for patients with severe mental disorders on a case-by-case basis, according to variables such as patient factors, environment, and systems in place in the medical facility. If a patient is already receiving treatment for a mental health disorder, the basic approach would be to allow the attending mental health clinician to continue treating the patient's illness. The medical team should observe the patient's condition in terms of their ability to tolerate the surgery [54]. The medical team should respond in a unified manner for each patient, focusing on extent to which symptoms are currently properly managed, regardless of the diagnosis. It is necessary to consider the treatment direction and support system on a case-by-case basis by referring to the patient referral documentation provided by the attending mental health clinician [54]. In some cases, it may be necessary to prioritize the stabilization of the patient's mental health while keeping the surgery plan on hold.

\section{Intraoperative and postoperative management}

\section{Challenges in the surgery for patients with severe obesity}

4.10. Airway and pharmacokinetics management and securing the surgical field are some of the difficulties associated with surgical procedures performed on patients with severe obesity.

It is often more challenging to manage the airways of patients with severe obesity (mask ventilation, endotracheal intubation) than those of non-obese patients. The larger the neck's circumference, the more difficult it is to perform direct observations using a laryngoscope. Other problems include restricted flexion/extension of the cervical spine, restriction of mouth opening, and enlarged soft tissue in the upper respiratory tract [147-152]. Moreover, the pharmacokinetics of fat-soluble anesthetics change. In laparoscopic surgery, it is necessary to keep the pneumoperitoneum pressure relatively high to secure the surgical field, which may cause an increase in intrathoracic pressure and a decrease in functional residual capacity, pneumothorax, and extraperitoneal emphysema. In addition, in patients with severe obesity, it is difficult to secure a surgical field due to the thick body wall, excessive accumulation of visceral fat, and hepatomegaly $[153,154]$.

\section{Perioperative complications of metabolic surgery}

\section{Intraoperative complications}

4.11. Although intraoperative complications are relatively rare, they increase the risk of incidence of postoperative complications

According to reports from large databases in Europe and the US, the prevalence of intraoperative complications is relatively low, ranging from 0.69 to $5.0 \%$ [155-157]. The incidence rates vary depending on the surgical technique (complexity of surgery), with complications seen in 7.3\% of open Roux-en Y gastric bypass procedures, 5.5\% of LRYGB procedures, and $3.0 \%$ of LAGB procedures [155]. The incidence rate of surgical complications in LSG was lower than that in LRYGB and higher than that in LAGB [158]. The incidence of complications in procedures that involve conversion from laparoscopic to open surgery is a risk factor for serious postoperative complications such as pulmonary embolism and myocardial infarction [155, 156]. Representative intraoperative complications include anesthesia-related complications, malfunctioning surgical instruments, intestinal injuries, liver injuries, gastrointestinal anastomosisrelated complications, spleen injuries, and major blood vessel injuries [155].

\section{Early postoperative complications}

4.12. Early postoperative complications are relatively rare. Major complications include anastomotic leakage, hemorrhage, deep vein thrombosis, pulmonary embolism, and cardiopulmonary complications (pneumonia, respiratory failure)

Early postoperative complications include anastomotic leakage, hemorrhage, deep vein thrombosis, pulmonary embolism, and cardiopulmonary complications (pneumonia, respiratory failure) [159-171]. According to reports from large databases in Europe and the US, the incidence rate of major early postoperative complications ranges from 0.2 to $10 \%$, and it is affected by comorbidities, surgical techniques, surgical methods (open or laparoscopic surgery), and the surgeon's skill level [157, 162, 163, 172-175]. In a multicenter retrospective study in Japan analyzing 831 patients (501, 149, 100, and 81 patients who underwent LSG, LSGDJB, LRYGB, and LAGB, respectively), early postoperative complications occurred in 77 (9.3\%) patients within 30 days after surgery [44]. 


\section{Operative mortality}

4.13. Operative mortality is relatively rare. A major cause of surgery-related deaths include pulmonary embolism, anastomotic leakage, cardiovascular events, and sepsis.

Risk factors connected to surgery-related deaths include the surgical procedure, degree of obesity, comorbidities (such as chronic obstructive pulmonary disease, ischemic heart failure, and state of needing long-term care), surgeon's skill level, surgical infrastructure, and the number of cases managed by the surgeon [154]. Mortality rates both during surgery and during hospitalization (after surgery) are generally low, at $<1 \%[166,176-179]$.

\section{Prevention of perioperative complications}

4.14. Sufficient preoperative assessment and preoperative management of comorbidities (such as diabetes, hypertension, NAFLD, and OSAS) and anesthesia risks are recommended to prevent perioperative complications, as is prohibition of smoking. (recommendation)

Patients with severe obesity have multiple obesityrelated health disorders, and it is important to evaluate and manage them before surgery. Risk factors of perioperative complications include male sex, BMI $\geq 50 \mathrm{~kg} / \mathrm{m}^{2}$, history of thrombosis, OSAS, combined metabolic abnormalities, and age $>50$ years $[180,181]$. Being bedridden and requiring long-term care are high-risk factors for complications [182]. In addition to undertaking CPAP therapy for OSAS, glycemic control, and a weight loss program before surgery, as mentioned previously, patients should also be instructed to refrain from smoking. Smoking is related to poor wound healing, anastomotic ulcers, and increased respiratory complications. Furthermore, smoking 20 cigarettes a day is considered equal to the prognosis of mild-to-moderate OSAS [183]. The patient should ideally stop smoking at least 6 weeks before metabolic surgery [183]. The Enhanced Recovery After Surgery (ERAS ${ }^{\circledR}$ ) program is used in the perioperative management of many surgical procedures, given its effectiveness for reducing complications, speeding up recovery, and shortening the inpatient period. Its utility for metabolic surgery has also been reported [184].

\section{Follow-up}

\section{Nutritional management}

4.15. Regular follow-up is recommended after surgery, because there are concerns about the deterioration of the patient's nutritional status due to reduced caloric, protein, vitamin, and mineral intake. (recommendation)

4.16. In addition to protein intake, patients are recommended to use supplements to replenish vitamins and minerals. (recommendation)

4.17. Because mean volumes and contents change significantly over time after surgery, it is recommended that patients receive nutritional intervention which fits the postoperative clinical course. (recommendation)

After metabolic surgery, the quantity of meal intake decreases with the reduced stomach volume. Many reports indicate that the caloric intake will reduce drastically down to $400-600 \mathrm{kcal} / \mathrm{day}$, immediately after surgery, and goes back up to $1000-1200 \mathrm{kcal} /$ day by one year after surgery, up further to $1200-1500 \mathrm{kcal} /$ day 3 years or more after surgery, and in the long-run, and become stable at the range of between $1 / 3$ to $1 / 2$ of the quantity of the meal intake before surgery [184-186]. It is also important to take the minimum necessary nutrients by consuming a limited amount of food after surgery to avoid deterioration in nutritional status.

After surgery, in addition to the absolute decrease in dietary intake, patients often experience changes in their food preferences such as difficulty in eating meat [187-190], which tends to result in reduced protein intake. This can lead to malnutrition (hypoalbuminemia), muscle weakness, anemia, hair loss, and nail cracking [89, 191, 192]. After surgery, the required protein intake varies, depending on reports and the surgical procedure used, but it is recommended that the patient ingests $60-120 \mathrm{~g} /$ day of protein. Reports on Japanese patients indicate that average protein intake may be insufficient for a certain period after surgery, it is necessary to be aware of this issue [186]. We propose dietary content that takes into consideration food preferences. It would be ideal to use supplements such as protein drinks, protein jellies, and formula diets as necessary to satisfy the required protein intake [193]. In addition, frequent vomiting may cause malnutrition (hypoalbuminemia), electrolyte imbalance, and nutritional deficiencies such as vitamin $\mathrm{B}_{1}$ deficiency and anemia [194, 195]. It is important to guide patients on how to eat, on chewing slowly, on choosing foods easy to eat, and on ways to cook the food. It is also necessary to be careful to avoid insufficient supplement intake, which can often cause nutrition deficiency [89].

Nutritional assessment, diagnosis, intervention, and monitoring following the surgical course are important parts of postoperative nutrition management. In terms of diet, the period from surgery completion to one month after surgery is regarded as the "Recovery/Transition Period." During this period, patients consume nutrients to recover from the surgery and prepare to transition from liquid food to solid food. Patients should ideally eat liquid and semi-solid food for up to 1 month after surgery, and it should be ensured that they 
drink water (at least 2 L/day) and ingest supplements and proteins (protein drinks). In particular, patients with severe obesity often have reduced kidney function already before surgery due to obesity-related kidney disease or diabetic nephropathy, and therefore, it is necessary to be careful to avoid dehydration. Make sure to keep track of the amount of water intake drunk by the patient. In some situations, it is recommended to provide oral rehydration solutions to the patient. The period from 1 month after surgery to 1 year after surgery is regarded as the "Weight Loss Period." It would be favorable to change the patient's diet gradually toward solid food from around one month after surgery. During this period, the patient should be monitored for protein intake (target: $65 \mathrm{~g} /$ day for men, $50 \mathrm{~g} /$ day or more for women), supplement intake, water intake (target: $2 \mathrm{~L} /$ day or more, including food), and proper snacking and drinking habits. It is necessary to ask the patient about their eating habits and perform nutritional intervention rather than focus only on the patient's body weight changes [45]. Around 1 year after surgery, the patient will enter the "Maintenance Period," during which their body weight will almost stabilize. The goal of nutritional management during this period and onward is to maintain a healthy body weight. The patient should be monitored for protein intake, supplement intake, snacking and drinking habits, and dietary balance (salt and saturated fatty acids). While nutritional management is to be conducted according to the Dietary Reference Intakes for Japanese (2020 Edition) [196] and the requirement of each nutrient varies depending on the patient, it is necessary to evaluate these individual levels by asking the patient questions and to gain a thorough understanding of their dietary and living conditions rather than fixate on the clinical laboratory test results. When the patient experiences rebounding weight gain, determine whether it is caused by a morphological issue associated with the surgical procedure (such as gastric dilation and dilation of anastomotic site) or should be dealt with by improvement of lifestyle habits, such as reviewing dietary contents. If the former is the main issue, re-operation should be considered. When the latter is the case, inadequate protein intake, snacking habits, and consumption of soft drinks are reported to adversely affect weight loss effect of the surgery [51, 194]. With regard to alcohol consumption, patients should be encouraged to drink in moderation, because the risk of alcoholism increases after surgery [197]. However, rather than impose a blanket restriction on all snacking and drinking, the patient's situation should be evaluated comprehensively to identify suitable eating habits.

Patients need to be evaluated comprehensively for the matters mentioned above and should receive long-term nutritional management. Continued intervention by staff familiar with metabolic surgery would help prevent postoperative complications and rebound weight gain.
Medical management of T2D, hypertension, dyslipidemia, OSAS, NAFLD, anemia and osteoporosis

4.18. Long-term follow-up of general management is recommended after surgery. (recommendation)

4.19. While glycemic control improves in most of diabetic patients after surgery, their diabetes medication should be adjusted when glycemic control deteriorates during long-term observation after surgery. (recommendation)

4.20. Dyslipidemia and hypertension improve with surgery, but their clinical remission rates are lower than those of diabetes. Therefore, continued management should be required. (recommendation)

4.21. Consider evaluation using PSG to judge whether weaning of CPAP therapy, even if OSAS may improve after surgery. (consideration)

The long-term therapeutic outcome of metabolic surgery depends on the patient's understanding of physical activity, eating behavior, and supplements usage [198]. To promote an adequate understanding, long-term follow-up and collaboration by a multidisciplinary medical team, including surgeons, physicians and psychiatrist, is important [199].

Each examination should involve a general follow-up and lifestyle guidance, based on blood tests and urinalysis, patient body weight, blood pressure as well as the assessment of mental health, physical activity, and sleep condition [200-202]. Pharmacotherapy should be conducted as necessary.

Meta-analysis/systematic review of 16 studies showed diabetes remission rate of $38.2-100 \%$ in cases after metabolic surgery [35]. Japanese reports also suggest that 76-85\% of patients undergoing LSG achieve clinical remission [44-47]. Still, it is necessary to select the appropriate medication for approximately $20 \%$ of patients who do not achieve clinical remission of diabetes. The STAMPEDE study reported the changes in diabetes medications 5 years after LSG. The ratio of patients using insulin decreased from 45 to $11 \%$ after surgery, and those who using 3 or more diabetes medications decreased from 19 to $13 \%$ after surgery [34]. In the case of 322 patients examined in J-SMART study, the ratios using oral diabetes medications and insulin dropped significantly from 57.7 to $9.6 \%$ and from 16.3 to $1.0 \%$, respectively, 2 years after LSG [45]. As some of the patients show gradual deteriorations of glycemic control, measurement of $\mathrm{HbA} 1 \mathrm{c}$ is preferred in each examination. Although clinical evidence is insufficient at present, metformin, SGLT2 inhibitors, dipeptidyl peptidase-4 inhibitors, GLP-1 receptor agonists, sulfonylureas and combined use with insulin have been effective to achieve optimal perioperative glycemic control [203]. While metformin is the most common agent for diabetes even after surgery, some suggest 
that its use should be avoided during the 3-4 months after surgery, during the negative energy balance period [204]. Moreover, some small-scale studies reported that SGLT2 inhibitors helped improve weight loss and glycemic control [205]. Postoperative withdrawing from insulin or starting SGLT2 inhibitors occasionally causes diabetic ketoacidosis, suggesting that diabetes medication should be carefully considered after surgery [206]. As postprandial reactive hypoglycemia may occur after LSG [207], continuous blood glucose monitoring should be considered to use for preventing the hypoglycemic events [208]. If a patient has advanced diabetic retinopathy before surgery, the rapid improvement in blood glucose levels after surgery may aggravate the retinopathy. Therefore, the status of retinopathy should be closely monitored after surgery [209].

The postoperative remission rates of dyslipidemia and hypertension are relatively low compared to that of diabetes. In J-SMART study, while the ratio of patients taking hypolipidemic drugs and those of taking antihypertensive drugs both dropped from 42.2 to $13.3 \%$ and from 58.9 to $30.2 \%$, respectively, 2 years after LSG, it was necessary to monitor those conditions regularly, in some cases with continuing medication [45]. Uric acid level decreases after metabolic surgery $[45,210]$ but could be elevated in the early stages after surgery. Some reports suggest that hypothyroidism might be developed after metabolic surgery [211]. Even vitamin $\mathrm{B}_{1}$ and vitamin $\mathrm{B}_{12}$ deficiencies are rarely occurred after LSG [212, 213], those vitamin levels are preferred to evaluate every 6-12 months. Moreover, $45-52 \%$ of patients show iron deficiency after surgery and around $15 \%$ develop anemia, suggesting the requirement of regularly follow-up by blood tests [214].
Many reports suggest that metabolic surgery leads to clinical improvement in OSAS [215]. In Japanese study, the prevalence of OSAS decreased from $79.2 \%$ before surgery to $31.0 \% 2$ years after surgery [45]. Because the craniomaxillofacial morphology is an important cause of OSAS, independent of obesity, treatment decisions for OSAS should be made after evaluation of the severity by PSG, even if patients ask to stop CPAP therapy with postoperative weight loss [216].

Between 82.4 and $98.3 \%$ of patients with severe obesity facing metabolic surgery have NAFLD, and between 63.2 and $77.5 \%$ have non-alcoholic steatohepatitis [217, 218], and liver fibrosis markedly improves after surgery [218]. As the risk of cholelithiasis increases after metabolic surgery [219], regularly examination by abdominal ultrasonography would be preferred. Furthermore, since bone mass may reduce due to secondary hyperparathyroidism associated with vitamin D and calcium deficiency after metabolic surgery [220, 221], bone mineral density evaluation would be preferred every 2 years.

\section{Mental health management}

4.22. It is recommended that all postoperative patients are followed-up as long as possible considered psychosocial aspect by the medical team stuff (mainly physicians and surgeons) and, if necessary, mental health professional (psychiatrist, psychosomatic physician, licensed psychologist). (recommendation)

4.23. After surgery, patients with mental disorders should be followed-up by the medical team stuff (mainly physicians and surgeons) in collaboration with the attending

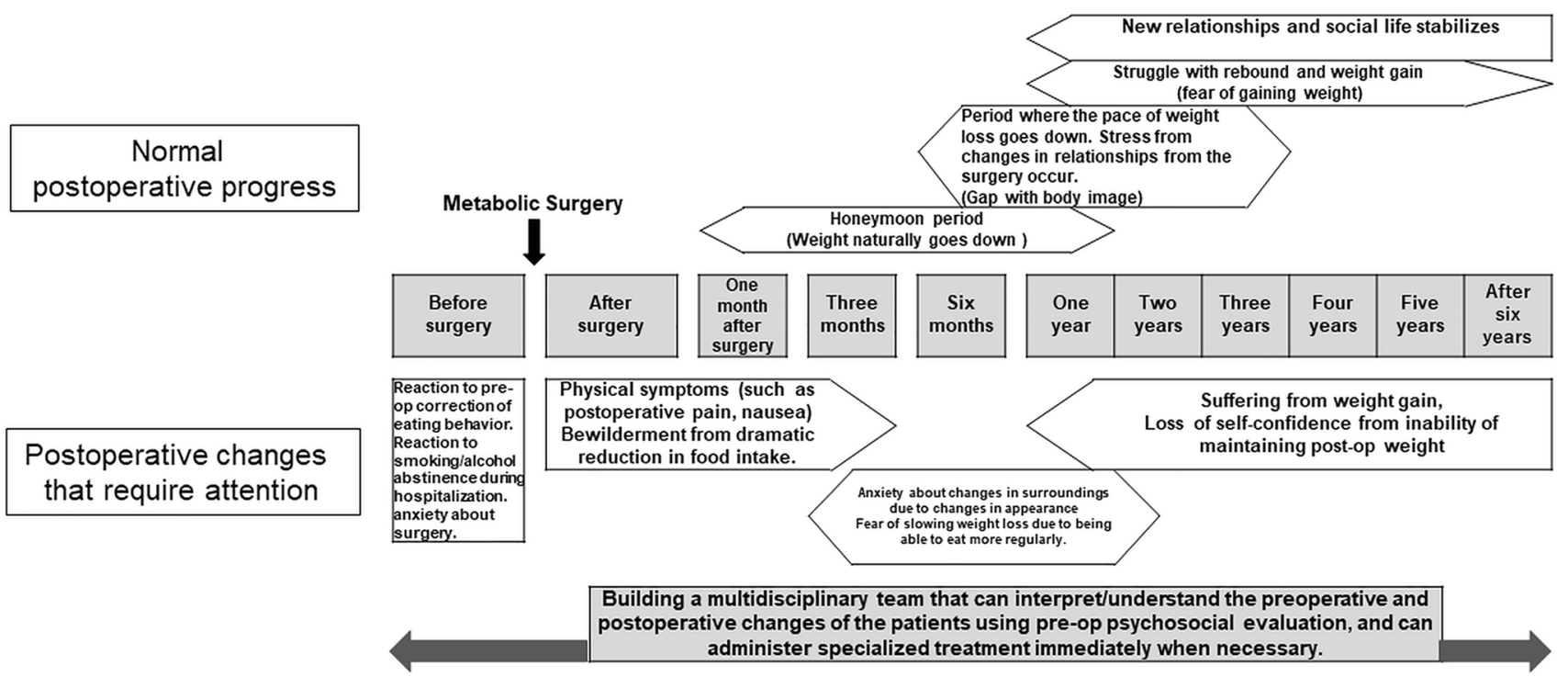

Fig. 3 Psychological changes observed after metabolic surgery 
mental health clinician (psychiatrist or psychosomatic physician), ascertaining the patient's condition. (recommendation)

4.24. The medical team stuff (mainly physicians and surgeons) must understand and keep in mind that patients can experience psychological changes due to changes in body weight and physical sensations after surgery, such as not being able to eat as expected. (consideration)

Figure 3 shows the psychological changes seen in patients after metabolic surgery [222, 223]. In general, the period up to 1 year after surgery is regarded as the "Honeymoon Period". Because this is the time during which patients experience their desired changes, such as weight loss, improvement of ease movement, improvement of physical appearance, and remission of physical illness. While most patients go through this period without major issues, from approximately 6 months to 1 year after surgery, another period comes in which they may feel confused by the decreasing speed of weight loss, and by changes to the way people interact with them because of their changing appearance and changes in human relationships as a consequence. After more time passes, albeit with some anxiety and fear about rebound weight regain, patients begin to build new human connections and become engaged in a more stable social life. The medical team stuff (mainly physicians and surgeons) should inform patients that most of them will experience such psychosocial changes after surgery, and should also be receptive to the patient's worries, support the positive efforts made by them to undergo treatment, and reassure them that they are on track to improve their condition (general psychotherapy). In this way, the medical team stuff (mainly physicians and surgeons) who are not mental health professionals can provide the necessary psychological support.

However, patients with mental disorders, can react negatively, as shown in the diagram. First, immediately before surgery, patients can react negatively to restriction on eating and prohibitions on smoking and drinking. They may also complain of surgery-related anxiety and express doubts and confusion about surgical pain, nausea, and drastic food intake reduction. Patients may also notice that symptoms similar to general postoperative symptoms manifest earlier and that they can increasing eating little by little, from around 6 months after surgery. They may express anxiety and frustration about the slowing speed of weight loss. If the patient experiences a rebound weight regain more than 1 year after surgery, they could lose self-confidence for their failure to maintain weight loss after surgery. These feelings could ultimately pose an obstacle to building new human relationships and living a more stable social life. As such, every stage of recovery presents risks for aggravation of mental health symptoms. Research in Europe and the US points to patients facing various problems after surgery, such as the increased risk of alcohol or drug abuse, smoking [224], increased risk of self-harm and suicide [225-227], and mental disorders that may emerge after surgery such as depression and anxiety disorders [228]. Furthermore, because surgery shrinks the stomach, patients become incapable of eating large amounts of food, which often result in grazing, a common cause of weight regain [229]. It has also been pointed out that patients with mental disorders tend to polarize into two groups after surgery: those without any drastic weight loss and those with excessive weight loss [51]. Therefore, it is recommended that the medical team stuff (mainly physicians and surgeons) simulate these postoperative changes such as psychiatric symptoms and eating behavior changes after surgery $[226,230]$. And the patients should be followed-up as long as possible considered psychosocial aspect by the medical team stuff, and if necessary, immediately mental health professional (psychiatrist, psychosomatic physician, licensed psychologist) [231].

\section{Exercise therapy}

4.25. Patients need to increase their daily physical activity levels as much as possible before surgery and improve their exercise tolerance to prevent postoperative muscle loss. (consideration)

4.26. Postoperative exercise therapy reduces fat mass without lean body mass loss. Therefore, it is recommended that patients increase physical activity levels. (recommendation)

4.27. Postoperative exercise therapy includes aerobic exercise, resistance training, and stretching. The intensity and duration of training should be increased gradually, within the patient's pain-free limits. (consideration)

Post-metabolic surgery exercise therapy reduces fat and increases physical activity levels without loss of lean body mass [232]. After surgery, muscle mass tends to decrease as caloric intake decreases. Therefore, patients undergoing metabolic surgery should increase their daily physical activity levels before surgery and continue after increasing exercise tolerance and maintaining muscle mass [233]. There is on report on improving the physical activity levels after surgery by starting exercise therapy before surgery [234]. However, for many patients with severe obesity, exercise therapy can be challenging, because they are affected by locomotor disorders. In these situations, patients can be motivated to increase their physical activity level by the suggestion that they sit less and stand more and do more day-to-day work, rather than conventional exercise therapy. Even if a patient can practice exercise 
therapy, physicians should be flexible by checking each patient's physical condition and exercise preferences [7].

The ASMBS guidelines state that patients should commence postoperative physical activities from the day of surgery. However, in many facilities in Japan, patients are instructed to get out of bed a day after surgery. The walking distance is gradually increased during the early stages after surgery. Patients practice each exercise to their painfree limit within 2 weeks after surgery. Subsequently, each exercise's intensity and duration are increased gradually in the following 4-6 weeks after surgery. Patients should avoid exercising the abdominal muscles until 8-12 weeks after surgery. The stepwise increase in exercise intensity and duration from before surgery to after surgery is outlined [235]. While Japan does not approve insurance coverage for exercise therapy guidance and physical activities, we hope improvements will be made in this direction.

Supplementary Information The online version contains supplementary material available at https://doi.org/10.1007/s13340-021-00551-0.

Acknowledgements We appreciate the special assistance given by Dr. Akira Umemura and the office staff of the JSTO in support of this work.

\section{Funding None.}

\section{Declarations}

Conflict of interest The Joint Committee of Japanese Society for Treatment of Obesity, The Japan Diabetes Society, and Japan Society for the Obesity have received reports of conflicts of interest over the past three years based on the following criteria regarding the economic relationship between members or collaborators and companies. Conflict of interest disclosure items: If applicable, declare the specific company names (organization names). If not applicable, not indicated. (1) Whether or not a member or an advisor of the board of directors of a company or an organization for profit is appointed, and the amount of remuneration ( 1 million yen or more per year from one company or organization for profit). (2) Holding of stocks and profits from those stocks (if one company's annual profit is 1 million yen or more, or holding 5\% or more of total issued stocks). (3) Patent royalties paid by companies or organizations for profit (one patent royalties is 1 million yen or more per year). (4) The value, lecture fees, etc. paid by companies or commercial organizations for the time and effort required for attending meetings (presentations, advice, etc.) or research (total fee from one company or organization for profit is 0.5 million yen or more per year). (5) Fee paid for writing, such as brochures paid by companies or organizations for profit (total fee from one company or organization for profit is 0.5 million yen or more per year). (6) The total amount of research funds that researchers can decide to apply by themselves for medical research such as joint research, contract research, clinical trials, etc. is 1 million yen or more per year from one company or organization for profit. (7) The total amount of donation for research that researchers can decide to apply provided by themselves for research fields/groups/departments is 1 million yen or more per year from one company or organization for profit. (8) If the researchers belong to a group/department with funding by a company donation, etc. (9) Receiving a favor such as private travel fee, gifts that are not directly related to research (total remuneration is 50,000 yen or more per year, from one company or organization). Akira Sasaki: (4) Covidien
Japan Inc. (6) ONO PHARMACEUTICAL CO., LTD., AstraZeneca K.K., NOVARTIS PHARMA K.K., Otsuka Pharmaceutical Co. Ltd., TAIHO PHARMACEUTICAL CO., LTD., Chugai Pharmaceutical Co., Ltd., PPD-SNBL K.K. (7) Covidien Japan Inc., Kaken Pharmaceutical Co.,Ltd. Koutaro Yokote: (4) Astellas Pharma Inc., AstraZeneca K.K., MSD K.K., Kowa Pharmaceutical Co., Ltd., NIPPON BOEHRINGER INGELHEIM CO., LTD., ONO PHARMACEUTICAL CO., LTD., NOVO NORDISK PHARMA LTD, TAKEDA PHARMACEUTICAL COMPANY LIMITED, Sumitomo Dainippon Pharma Co., Ltd., NOVARTIS PHARMA K.K., Mitsubishi Tanabe Pharma Corporation, ELI LILLY JAPAN K.K., TAISHO PHARMACEUTICAL CO., LTD., Sanofi K.K., Janssen Pharmaceutical K.K., DAIICHI SANKYO CO., LTD (6) TAISHO PHARMACEUTICAL CO., LTD. (7) ONO PHARMACEUTICAL CO., LTD., TAKEDA PHARMACEUTICAL COMPANY LIMITED, MSD K.K., DAIICHI SANKYO CO., LTD, Mitsubishi Tanabe Pharma Corporation, Shionogi \& Co., Ltd., Sumitomo Dainippon Pharma Co., Ltd., NIPPON BOEHRINGER INGELHEIM CO., LTD., TEIJIN PHARMA LIMITED, BAYER YAKUHIN, LTD., Astellas Pharma Inc., Kowa Company, Ltd., NOVO NORDISK PHARMA LTD, TAISHO PHARMACEUTICAL CO., LTD. Takeshi Naitoh: (4) Johnson \& Johnson K.K,Covidien Japan Inc.,Sumitomo Bakelite Co., Ltd.,Terumo Corporation,MSD K.K. (7) Chugai Pharmaceutical Co., Ltd. TAIHO PHARMACEUTICAL CO., LTD.,Covidien Japan Inc. Junji Fujikura: None to disclose. Karin Hayashi: None to disclose. Yushi Hirota: (4) ELI LILLY JAPAN K.K., Sanofi K.K. Nobuya Inagaki: (4) Kowa Pharmaceutical Co., Ltd., MSD K.K., Astellas Pharma Inc., NOVO NORDISK PHARMA LTD, ONO PHARMACEUTICAL CO., LTD., NIPPON BOEHRINGER INGELHEIM CO., LTD., TAKEDA PHARMACEUTICAL COMPANY LIMITED, Mitsubishi Tanabe Pharma Corporation, Sumitomo Dainippon Pharma Co., Ltd., Sanofi K.K., ELI LILLY JAPAN K.K. (6) Terumo Corporation, Drawbridge Inc, asken. (7) TAKEDA PHARMACEUTICAL COMPANY LIMITED, MSD K.K., ONO PHARMACEUTICAL CO., LTD., Mitsubishi Tanabe Pharma Corporation, KISSEI PHARMACEUTICAL CO., LTD, Sanofi K.K., DAIICHI SANKYO CO., LTD, Japan Tobacco Inc., Kyowa Kirin Co., Ltd., Sumitomo Dainippon Pharma Co., Ltd., Astellas Pharma Inc., SANWA KAGAKU KENKYUSHO, Nippon Boehringer Ingelheim Co., Ltd., Novo Nordisk Pharma Ltd., Novartis Pharma K.K., Life Scan Japan (8).TAKEDA PHARMACEUTICAL COMPANY LIMITED, MSD K.K., ONO PHARMACEUTICAL CO., LTD., Mitsubishi Tanabe Pharma Corporation. Yasushi Ishigaki: (4) MSD K.K., NOVO NORDISK PHARMA LTD, Sanofi K.K., TAKEDA PHARMACEUTICAL COMPANY LIMITED, ONO PHARMACEUTICAL CO., LTD., NOVARTIS PHARMA K.K., Kowa Pharmaceutical Co., Ltd., BAYER YAKUHIN, LTD. (6) DAIICHI SANKYO CO., LTD, TAKEDA Science Foundation (7) ONO PHARMACEUTICAL CO., LTD., MSD K.K. Kazunori Kasama: (4) Johnson \& Johnson K.K., Covidien Japan Inc. (6) Covidien Japan Inc., Nikkiso Co., Ltd. Eri Kikkawa: None to disclose. Hidenori Koyama: None to disclose. Hiroaki Masuzaki: None to disclose. Takeshi Miyatsuka: (8) MSD K.K., Mitsubishi Tanabe Pharma Corporation, Nippon Boehringer Ingelheim Co., Ltd., ONO PHARMACEUTICAL CO., LTD., Kowa Company, Ltd. Takehiro Nozaki: None to disclose. Wataru Ogawa: (4) Sumitomo Dainippon Pharma Co., Ltd., NOVARTIS PHARMA K.K., Nippon Boehringer Ingelheim Co., Ltd., TAKEDA PHARMACEUTICAL COMPANY LIMITED, Mitsubishi Tanabe Pharma Corporation, Abbott Japan LLC. (6) Noster,Nippon, Boehringer Ingelheim Co., Ltd., Boehringer Ingelheim Pharma GmbH\&Co.KG, ELI LILLY JAPAN K.K., NOVO NORDISK PHARMA LTD, Abbott Japan LLC., Abbott Diabetes Care UK Ltd, Sumitomo Dainippon Pharma Co., Ltd. (7) Kowa Company, Ltd., NOVO NORDISK PHARMA LTD, Astellas Pharma Inc., Sumitomo Dainippon Pharma Co., Ltd., ONO PHARMACEUTICAL CO., LTD., TAKEDA PHARMACEUTICAL COMPANY LIMITED, Abbott Japan LLC., NOVARTIS PHARMA K.K., DAIICHI SANKYO CO., LTD, ELI LILLY JAPAN K.K., Mitsubishi 
Tanabe Pharma Corporation, Nippon Boehringer Ingelheim Co., Ltd. Masayuki Ohta: None to disclose. Shinichi Okazumi: None to disclose. Michio Shimabukuro: (4) Kowa Company, Ltd., NIPPON BOEHRINGER INGELHEIM CO., LTD., Sanofi K.K., SANWA KAGAKU KENKYUSHO, TAKEDA PHARMACEUTICAL COMPANY LIMITED, ELI LILLY JAPAN K.K., Mitsubishi Tanabe Pharma Corporation, MSD K.K., NOVO NORDISK PHARMA LTD, Astellas Pharma Inc., NOVARTIS PHARMA K.K., DAIICHI SANKYO CO., LTD. Iichiro Shimomura: (4) MSD K.K., ONO PHARMACEUTICAL CO., LTD., Kowa Company, Ltd., TAISHO PHARMACEUTICAL CO., LTD., TAKEDA PHARMACEUTICAL COMPANY LIMITED, ELI LILLY JAPAN K.K., NOVO NORDISK PHARMA LTD. (6) Kowa Company, Ltd., Kobayashi Pharmaceutical Co., Ltd., ROHTO Pharmaceutical Co.,Ltd. (7)Kyowa Kirin Co., Ltd., Kowa Company, Ltd., Sanofi K.K., DAIICHI SANKYO CO., LTD, Sumitomo Dainippon Pharma Co., Ltd., TAKEDA PHARMACEUTICAL COMPANY LIMITED, Mitsubishi Tanabe Pharma Corporation, TEIJIN PHARMA LIMITED, Novo Nordisk Pharma Ltd., MOCHIDA PHARMACEUTICAL CO., LTD. Hitoshi Nishizawa: None to disclose. Atsuhito Saiki: None to disclose. Yosuke Seki: (4) Johnson \& Johnson K.K, Covidien Japan Inc. (6) Covidien Japan Inc., Nikkiso Co., Ltd, Sunny Health Co.,Ltd., Daiwa Securities Health Foundation. Nobuhiro Shojima: None to disclose. Motoyoshi Tsujino: None to disclose. Satoshi Ugi: None to disclose. Hirotaka Watada: (4) Mitsubishi Tanabe Pharma Corporation, Sumitomo Dainippon Pharma Co., Ltd., SANWA KAGAKU KENKYUSHO, TAKEDA PHARMACEUTICAL COMPANY LIMITED, Sanofi K.K., Kowa Company, Ltd., MSD K.K., Nippon Boehringer Ingelheim Co., Ltd., Eli Lilly Japan K.K., Novo Nordisk Pharma Ltd., AstraZeneca K.K., ONO PHARMACEUTICAL CO., LTD., Astellas Pharma Inc., Kyowa Kirin Co., Ltd., Terumo Corporation. (6) Sanofi K.K., Kowa Company, Ltd., Nippon Boehringer Ingelheim Co., Ltd., Yakult Honsha Co.,Ltd. (7) TAKEDA PHARMACEUTICAL COMPANY LIMITED, NIPPON BOEHRINGER INGELHEIM CO., LTD., KISSEI PHARMACEUTICAL CO., LTD, NOVO NORDISK PHARMA LTD, Mitsubishi Tanabe Pharma Corporation, LifeScan Japan, Kyowa Kirin Co., Ltd., Sumitomo Dainippon Pharma Co., Ltd., TAISHO PHARMACEUTICAL CO., LTD., Abbott Japan LLC., DAIICHI SANKYO CO., LTD, Astellas Pharma Inc., ONO PHARMACEUTICAL CO., LTD., TEIJIN PHARMA LIMITED, Sanofi K.K., MSD K.K., Pfizer Japan Inc. (8)TAKEDA PHARMACEUTICAL COMPANY LIMITED, Soiken Inc., SANWA KAGAKU KENKYUSHO, Sumitomo Dainippon Pharma Co., Ltd., TAISHO PHARMACEUTICAL CO., LTD., Mitsubishi Tanabe Pharma Corporation, ONO PHARMACEUTICAL CO., LTD., Kowa Pharmaceutical Co., Ltd., MSD K.K.,Nippon Boehringer Ingelheim Co., Ltd. Toshimasa Yamauchi: (4) Astellas Pharma Inc., AstraZeneca K.K., ONO PHARMACEUTICAL CO., LTD., Sanofi K.K., TAKEDA PHARMACEUTICAL COMPANY LIMITED, DAIICHI SANKYO CO., LTD, NOVARTIS PHARMA K.K., NOVO NORDISK PHARMA LTD. (6) AstraZeneca K.K., Kowa Company, Ltd., MSD K.K., DAIICHI SANKYO CO., LTD, Sanofi K.K., NIPPON BOEHRINGER INGELHEIM CO., LTD., Aero Switch,SANWA KAGAKU KENKYUSHO, Minophagen Pharmaceutical Co., LTD., Mitsubishi Corporation Life Sciences Limited., NIPRO CORPORATION. (7) NOVO NORDISK PHARMA LTD, Mitsubishi Tanabe Pharma Corporation, TAISHO PHARMACEUTICAL CO., LTD., KISSEI PHARMACEUTICAL CO., LTD, Kyowa Kirin Co., Ltd., DAIICHI SANKYO CO., LTD. (8) TAKEDA PHARMACEUTICAL COMPANY LIMITED, ONO PHARMACEUTICAL CO., LTD., NOVO NORDISK PHARMA LTD, Mitsubishi Tanabe Pharma Corporation, MSD K.K., NIPPON BOEHRINGER INGELHEIM CO., LTD., Kowa Company, Ltd., Asahi Mutual Life Insurance Company.Takashi Yamaguchi: None to disclose. Ichiro Tatsuno: (4) NOVARTIS PHARMA K.K., TAKEDA PHARMACEUTICAL COMPANY LIMITED. (7) BAYER YAKUHIN, LTD., TAKEDA PHARMACEUTICAL COMPANY LIMITED, ONO PHARMACEUTICAL CO., LTD. Kojiro Ueki: (4)
Sumitomo Dainippon Pharma Co, Ltd., MSD K.K., Kyowa Kirin Co., Ltd., DAIICHI SANKYO CO.,LTD., NIPPON BOEHRINGER INGELHEIM CO.,LTD., TAKEDA PHARMACEUTICAL COMPANY LIMITED, NOVO NORDISK PHARMA LTD, Mitsubishi Tanabe Pharma Corporation, AstraZeneca K.K., ONO PHARMACEUTICAL CO., LTD., Sanofi K.K., Astellas Pharma Inc. (6) Astellas Pharma Inc., NOVO NORDISK PHARMA LTD., ELI LILLY JAPAN K.K., NIPPON BOEHRINGER INGELHEIM CO., LTD., Abbott Japan LLC., MSDK.K. (7) Sanofi K.K., NIPPON BOEHRINGER INGELHEIM CO., LTD., NOVO NORDISK PHARMA LTD, Sumitomo Dainippon Pharma Co., Ltd., Kyowa Kirin Co., Ltd., TAKEDA PHARMACEUTICAL COMPANY LIMITED, Mitsubishi Tanabe Pharma Corporation, Astellas Pharma Inc., DAIICHI SANKYO CO., LTD, ONO PHARMACEUTICAL CO., LTD. Takashi Kadowaki: (4) MSD K.K., Astellas Pharma Inc., AstraZeneca K.K., Abbott Japan LLC., Sanofi K.K., Terumo Corporation, NOVO NORDISK PHARMA LTD, ONO PHARMACEUTICAL CO., LTD., Mitsubishi Tanabe Pharma Corporation, ELI LILLY JAPAN K.K., NIPPON BOEHRINGER INGELHEIM CO., LTD., TAKEDA PHARMACEUTICAL COMPANY LIMITED, Sumitomo Dainippon Pharma Co., Ltd. (5) TAKEDA PHARMACEUTICAL COMPANY LIMITED. (6) AstraZeneca K.K., DAIICHI SANKYO CO., LTD, TAKEDA PHARMACEUTICAL COMPANY LIMITED. (7) Astellas Pharma Inc., KISSEI PHARMACEUTICAL CO., LTD, Sanofi K.K., NOVO NORDISK PHARMA LTD, ONO PHARMACEUTICAL CO., LTD., Taisho Pharma Co., Ltd., DAIICHI SANKYO CO., LTD, Mitsubishi Tanabe Pharma Corporation, TAKEDA PHARMACEUTICAL COMPANY LIMITED, Sumitomo Dainippon Pharma Co., Ltd. (8) MSD K.K., NOVO NORDISK PHARMA LTD, Kowa Pharmaceutical Co., Ltd, ONO PHARMACEUTICAL CO., LTD., Mitsubishi Tanabe Pharma Corporation, NIPPON BOEHRINGER INGELHEIM CO., LTD., TAKEDA PHARMACEUTICAL COMPANY LIMITED, Asahi Mutual Life Insurance Company.

Ethical statement This article does not contain any studies with human or animal subjects performed by any of the authors.

\section{References}

1. World health organization. Obesity and overweight; 2020. https:// www.who.int/news-room/fact-sheets/detail/obesity-and-overw eight. Accessed 15 Sept 2021

2. Examination Committee of Criteria for 'Obesity Disease' in Japan; Japan Society for the Study of Obesity. New Criteria for 'Obesity Disease' in Japan. Circ J. 2002;66:987-92

3. Sjöström L, et al. Effects of bariatric surgery on mortality in Swedish obese subjects. N Engl J Med. 2007;357:741-52.

4. Cardoso L, et al. Short- and long-term mortality after bariatric surgery: a systematic review and meta-analysis. Diabetes Obes Metab. 2017;19:1223-32.

5. Syn NL, et al. Association of metabolic-bariatric surgery with long-term survival in adults with and without diabetes: a onestage meta-analysis of matched cohort and prospective controlled studies with 174772 participants. Lancet. 2021;397:1830-41.

6. Rubino F, Delegates of the 2nd Diabetes Surgery Summit, et al. Metabolic surgery in the treatment algorithm for type 2 diabetes: a joint statement by international diabetes organizations. Diabetes Care. 2016;39:861-77.

7. Japan Society for the Study of Obesity. Guidelines for the management of obesity disease. Tokyo: Life Science Publishing; 2016. (in Japanese).

8. Japan Diabetes Society. Treatment guideline for diabetes 2020 2021. Tokyo: Bunkodo; 2020. (in Japanese) 
9. Japan Diabetes Society. Japanese Clinical Practice Guideline for Diabetes 2019. Tokyo: Nankodo; 2019. (in Japanese)

10. Montesi L, et al. Long-term weight loss maintenance for obesity: a multidisciplinary approach. Diabetes, Metabolic Syndrome And Obesity. Targets Therapy. 2016;9:27-46.

11. The Obesity Society and American College of Cardiology/ American Heart Association Task Force on Practice Guidelines. Executive summary: guidelines (2013) for the management of overweight and obesity in adults. Obesity. 2014;22:S5-39.

12. Toyama T, et al. Effect of SGLT2 inhibitors on cardiovascular, renal and safety outcomes in patients with type 2 diabetes mellitus and chronic kidney disease: a systematic review and metaanalysis. Diabetes Obes Metab. 2019;21:1237-50.

13. Cai X, et al. The association between the dosage of SGLT2 inhibitor and weight reduction in type 2 diabetes patients: a metaanalysis. Obesity (Silver Spring). 2018;26:70-80.

14. Monami M, et al. Effects of glucagon-like peptide-1 receptor agonists on body weight: a meta-analysis. Exp Diabetes Res. 2012;2012:672658.

15. Potts JE, et al. The effect of glucagon-like peptide 1 receptor agonists on weight loss in type 2 diabetes: a systematic review and mixed treatment comparison meta-analysis. PLoS ONE. 2015;10: e0126769.

16. Izzi-Engbeaya C, et al. Adverse outcomes in COVID-19 and diabetes: a retrospective cohort study from three London teaching hospitals. BMJ Open Diab Res Care. 2021;9: e001858.

17. Anderson MR, et al. Body mass index and risk for intubation or death in SARS-CoV-2 infection: a retrospective cohort study. Ann Intern Med. 2020;173:782-90.

18. Shoar $\mathrm{S}$, et al. Long-term and midterm outcomes of laparoscopic sleeve gastrectomy versus roux-en-y gastric bypass: a systematic review and meta-analysis of comparative studies. Surg Obes Relat Dis. 2017;13:170-80.

19. Yan Y, et al. Roux-en-Y gastric bypass versus medical treatment for type 2 diabetes mellitus in obese patients: a systematic review and meta-analysis of randomized controlled trials. Medicine (Baltimore). 2016;95: e3462.

20. Ribaric G, et al. Diabetes and weight in comparative studies of bariatric surgery vs conventional medical therapy: a systematic review. Obes Surg. 2014;24:437-55.

21. Chang SH, et al. The Effectiveness and risk of bariatric surgery: an updated systematic review and meta-analysis, 2003-2012. JAMA Surg. 2014;149:275-87.

22. Mental Health Subcommittee in Japan Society for Treatment of Obesity. Understanding and dealing with psychosocial problems necessary for obesity treatment (ed). Tokyo: Compus; 2016. (in Japanese)

23. Hayashi K, et al. Psychiatric disorders with obesity patients: mainly depression. Jpn J Psychosom Int Med. 2016;20:267-72 (in Japanese).

24. Rubio F, et al. Joint international consensus statement for ending stigma of obesity. Nat Med. 2020;26:485-97.

25. Caterson ID, et al. Gaps to bridge: misalignment between perception, reality and actions in obesity. Diabetes Obes Metab. 2019;21:1914-24.

26. Japan Diabetes Society, Japan Association for Diabetes Education and Care Advocacy. Committee. 2021. http://www.jds.or. jp/modules/important/index.php?content_id=133. Accessed 15 Sept 2021 (in Japanese)

27. Locke AE, et al. Genetic studies of body mass index yield new insights for obesity biology. Nature. 2015;518:197-206.

28. Akiyama M, et al. Genome-wide association study identifies 112 loci for body mass index in the Japanese population. Nat Genet. 2017;49:1458-67.
29. Spracklen $\mathrm{CN}$, et al. Identification of type 2 diabetes loci in 433,540 East Asian individuals. Nature. 2020;582:240-5.

30. Kasama K, et al. IFSO-APC consensus statement 2011. Obes Surg. 2012;22:677-84.

31. American Diabetes Association. Obesity management for the treatment of type 2 diabetes: standards of medical care in diabetes-2020. Diabetes Care. 2020;43(Supplement 1):S89-97.

32. Treatment Guideline Committee for Bariatric Surgery in Japanese Society for Treatment of Obesity. Guidelines for safe and outstanding surgical treatment for severe obesity in Japan (2013 ed.). 2021. http://plaza.umin.ne.jp/ jsto/gakujyutsu/updata/surge ry_guideline_2013.pdf. Accessed 15 Sept 2021 (in Japanese)

33. Mingrone G, et al. Bariatric-metabolic surgery versus conventional medical treatment in obese patients with type 2 diabetes: 5 year follow-up of an open-label, single-centre, randomised controlled trial. Lancet. 2015;386:964-73.

34. Schauer PR, STAMPEDE Investigators, et al. Bariatric surgery versus intensive medical therapy for diabetes 3-year outcomes. N Engl J Med. 2014;370:2002-13.

35. Ribaric G, et al. Diabetes and weight comparative studies of bariatric surgery vs conventional medical therapy: a systematic review and meta-analysis. Obes Surg. 2014;24:437-55.

36. Carlsson LMS, et al. Life expectancy after bariatric surgery in the Swedish obese subjects study. N Engl J Med. 2020;383:1535-43.

37. Aminian A, et al. Association of metabolic surgery with major adverse cardiovascular outcomes in patients with type 2 diabetes and obesity. JAMA. 2019;322:1271-82.

38. Yan G, et al. Long-term outcomes of macrovascular diseases and metabolic indicators of bariatric patients with a meta-analysis. PLoS ONE. 2019;14: e0224828.

39. Sjöström L, et al. Association of bariatric surgery with longterm remission of type 2 diabetes and with microvascular and macrovascular complications. JAMA. 2014;311:2297-304.

40. Sheng B, et al. The long-term effects of bariatric surgery on type 2 diabetes remission, microvascular complications, and mortality: a systemic review and meta-analysis. Obes Surg. 2017;27:2724-32.

41. Upala $S$, et al. Bariatric surgery reduces urinary albumin exertion in diabetic nephropathy: a systemic review and meta-analysis. Surg Obes Relat Dis. 2016;12:1037-44.

42. Lindekilde $\mathrm{N}$, et al. The impact of bariatric surgery on quality of life: a systematic review and meta-analysis. Obes Rev. 2015;16:639-51.

43. Seki Y, et al. Long-term outcomes of laparoscopic sleeve gastrectomy in morbidly obese Japanese patients. Obes Surg. 2016;26:138-45.

44. Haruta $\mathrm{H}$, et al. Long-term outcomes of bariatric and metabolic surgery in Japan: results of a multi-institutional survey. Obes Surg. 2017;27:754-62.

45. Saiki A, et al. Background characteristics and postoperative outcomes of insufficient weight loss after laparoscopic sleeve gastrectomy in Japanese patients. Ann Gastroenterol Surg. 2019;26:638-47.

46. Naitoh T, et al. Efficacy of sleeve gastrectomy with duodenal-jejunal bypass for the treatment of obese severe diabetes patients in Japan: a retrospective multicenter study. Obes Surg. 2018;28:497-505.

47. Umemura A, et al. Prognostic factors and a new preliminary scoring system for remission of type 2 diabetes mellitus after laparoscopic sleeve gastrectomy. Surg Today. 2020;50:1056-64.

48. Saiki A, et al. Background characteristics and diabetes remission after laparoscopic sleeve gastrectomy in Japanese patients with type 2 diabetes stratified by BMI: subgroup analysis of J-SMART. Diabetol Int. 2021. https://doi.org/10.1007/ s13340-020-00487-x. 
49. Heymsfield SB, et al. Mechanisms, pathophysiology, and management of obesity. NEJM. 2017;376:254-66.

50. Simon GE, et al. Association between obesity and psychiatric disorders in the US adult population. Arch Gen Psychiatry. 2006;63:824-30.

51. Saiki A, et al. Impact of mental health background and nutrition intake on medium-term weight loss in Japanese patients undergoing laparoscopic sleeve gastrectomy. Obes Facts. 2020;13:371-83.

52. Sarwer DB, et al. Psychopathology, disordered eating, and impulsivity as predictors of outcomes of bariatric surgery. Surg Obes Relat Dis. 2019;15:650-5.

53. Yu Y, et al. Predictors of weight regain after sleeve gastrectomy: an integrative review. Surg Obes Relat Dis. 2019;15:995-1005.

54. Sogg S, et al. Recommendations for the presurgical psychosocial evaluation of bariatric surgery patients. Surg Obes Relat Dis. 2016;12:731-49.

55. Mechanick JI, et al. Clinical practice guidelines for the perioperative nutritional, metabolic, and nonsurgical support of the bariatric surgery patient: 2013 Update. Endocr Pract. 2013;19:e1-36.

56. Eldar S, et al. A focus on surgical preoperative evaluation of the bariatric patient - the Cleveland Clinic protocol and review of the literature. Surgeon. 2011;9:273-7.

57. Hout GCM, et al. Psychosocial predictors of success following bariatric surgery. Obes Surg. 2005;15:552-60.

58. Pull CB. Current psychological assessment practices in obesity surgery programs: what to assess and why. Curr Opin Psychiatry. 2010;23:30-6.

59. Fabricatore AN, et al. How do mental health professionals evaluate candidates for bariatric surgery? Survey results. Obes Surg. 2005;15:567-73.

60. Mechanick JI, et al. Clinical practice guidelines for the perioperative nutrition, metabolic, and nonsurgical support of patients undergoing bariatric procedures-2019 update. Endocr Pract. 2019;25:1346-59.

61. Wharton S, et al. Obesity in adults: a clinical practice guideline. CMAJ. 2020;192:E875-91.

62. Rubino F, et al. Joint international consensus statement for ending stigma of obesity. Nat Med. 2020;26:485-97.

63. Arora M, et al. Stigma and obesity: the crux of the matter. Lancet Public Health. 2019;4:E549-50.

64. Albury $\mathrm{C}$, et al. The importance of language in engagement between health-care professionals and people living with obesity: a joint consensus statement. Lancet Diabetes Endocrinol. 2020;8:447-55.

65. Spahlholz J, et al. Obesity and discrimination: a systematic review and meta-analysis of observational studies. Obes Rev. 2016;17:43-55.

66. Kaly P, et al. Unrealistic weight loss expectations in candidates for bariatric surgery. Surg Obes Relat Dis. 2008;4:6-10.

67. Lier HØ, et al. Psychiatric disorders and participation in pre- and postoperative counselling groups in bariatric surgery patients. Obes Surg. 2011;21:730-7.

68. Sawamoto R, et al. Predictors of successful long-term weight loss maintenance: a two-year follow-up. BioPsychoSoc Med. 2017;11:14

69. Dawes AJ, et al. Mental health conditions among patients seeking and undergoing bariatric surgery. A meta-analysis. JAMA. 2016;315:150-63.

70. Cassin S, et al. Psychometric properties of the Patient Health Questionnaire (PHQ-9) as a depression screening tool for bariatric surgery candidates. Psychosomatics. 2013;54:352-8.

71. Pinto TF, et al. Obesity, hypersomnolence, and quality of sleep: the impact of bariatric surgery. Obes Surg. 2017;27:1775-9.
72. Grupski AE, et al. Examining the binge eating scale in screening for binge eating disorder in bariatric surgery candidates. Obes Surg. 2013;23:1-6.

73. Oyama T, et al. High lambda style as pathognomonic personality of obesity patients: analysis with rorschach test. J Jpn Soc Study Obes. 2009;15:39-44.

74. Hayashi K, et al. Psychological characteristics of obesity patientsfrom the analysis of rorschach variables. Jpn J Psychosom Med. 2016;56:920-30.

75. Wimmelmann CL, et al. Association of personality with body mass index and obesity in a large late midlife community sample. Obes Facts. 2018;11:129-43.

76. Provost DA. Indications and contraindications for bariatric surgery. In: Nugyen NT, editor. The ASMBS textbook of bariatric surgery. 1st ed. New York: Springer; 2015. p. 73-6.

77. De Luca M, et al. Indications for surgery for obesity and weightrelated diseases: position statements from the International Federation for the Surgery of Obesity and Metabolic Disorders (IFSO). Obes Surg. 2020;26:1659-96.

78. Fried M, et al. Interdisciplinary European Guidelines On Metabolic And Bariatric Surgery. Obes Facts. 2013;6:449-68.

79. Flores CA. Psychological assessment for bariatric surgery. Arq Bras Cir Dig. 2014;27:59-62.

80. Clark MM, et al. Psychosocial factors and 2-year outcome following bariatric surgery for weight loss. Obes Surg. 2003;13:739-45.

81. Heinberg L, et al. History of substance abuse relates to improved postbariatric body mass index outcomes. Surg Obes Relat Dis. 2010;6:417-21.

82. Applegate KL, et al. Introduction to psychological consultations for bariatric surgery patients. In: Still C, Sarwer DB, Blankenship J, editors., et al., The ASMBS textbook of bariatric surgery. 2nd ed. New York: Springer; 2014. p. 33-42.

83. Peterhänsel $\mathrm{C}$, et al. Obesity and co-morbid psychiatric disorders as contraindications for bariatric surgery? Int J Surg Case Rep. 2014;5:1268-70

84. American Diabetes Association. Standards of medical care in diabetes-2010. Diabetes Care. 2010;33(Supplement 1):S11-61.

85. Melissas J. IFSO guidelines for safety, quality, and excellence in bariatric surgery. Obes Surg. 2008;18:497-500.

86. SAGES Guidelines Committee. SAGES guideline for clinical application of laparoscopic bariatric surgery. Surg Endosc. 2008;22:2281-300.

87. Japan Society for the Study of Obesity. Metabolic surgery accreditation facility review service. 2021. http://plaza.umin. ne.jp/ jsto/gekashisetsu/ninteishinsa2020.html. Accessed 15 Sept 2021 (in Japanese)

88. Requirements for the introduction of laparoscopic bariatric and diabetic surgery in the Japanese Society of Endoscopic Surgery and the Japanese Society for bariatric surgery. http:// plaza.umin.ne.jp/ jsto/about/pdf/dounyuyouken.pdf. Accessed 15 Sept 2021 (in Japanese)

89. Treatment Guideline Committee in Japanese Society for Treatment of Obesity. Comprehensive treatment guide for obesity disease. Compass publishing, 2013 (in Japanese)

90. Insurance Committee in Japanese Society for Treatment of Obesity. Urgent questionnaire Survey 2021. http://plaza.umin. ne.jp/ jsto/about/pdf/questionnairesurvey2021.pdf. Accessed 15 Sept 2021 (in Japanese)

91. Sasaki A, et al. Current status of bariatric surgery in Japan and effectiveness in obesity and diabetes. J Gastroenterol. 2014;49:57-63.

92. Kasama K, et al. Laparoscopic sleeve gastrectomy with duodenojejunal bypass: technique and preliminary results. Obes Surg. 2009; 19:1341-5. 
93. Cresci B, et al. Metabolic surgery for the treatment of type 2 diabetes: a network meta-analysis of randomized controlled trials. Diabetes Obes Metab. 2020;22:1378-87.

94. Cummings DE, et al. Bariatric/metabolic surgery to treat type 2 DIABETES in patients with a BMI $<35 \mathrm{~kg} / \mathrm{m}^{2}$. Diabetes Care. 2016;39:924-33.

95. Schauer PR, et al. Bariatric surgery versus intensive medical therapy for diabetes: 5-year outcomes. N Engl J Med. 2017;376:641-51.

96. Dang JT, et al. Predictive factors for diabetes remission after bariatric surgery. Can J Surg. 2019;62:315-9.

97. Capoccia D, et al. Long-term metabolic effects of laparoscopic sleeve gastrectomy. Obes Surg. 2018;28:2289-96.

98. Vidal J, et al. Type 2 diabetes mellitus and the metabolic syndrome following sleeve gastrectomy in severely obese subjects. Obes Surg. 2008;18:1077-82.

99. Capoccia D, et al. Weight regain and diabetes evolution after sleeve gastrectomy: a cohort study with over 5 years of followup. Obes Surg. 2020;30:1046-51.

100. Viscido G, et al. Obese patients with type 2 diabetes: outcomes after laparoscopic sleeve gastrectomy. J Laparoendosc Adv Surg Tech A. 2019;29:655-62.

101. Ramos-Levi AM, et al. Statistical models to predict type 2 diabetes remission after bariatric surgery. J Diabetes. 2014;6:472-7.

102. Ohira M, et al. Determinants of type 2 diabetes remission after bariatric surgery in obese Japanese patients: a retrospective cohort study. Diabetol Int. 2021. https://doi.org/10.1007/ s13340-021-00493-7.

103. Lee WJ, et al. Predicting success of metabolic surgery: age, body mass index, C-peptide, and duration score. Surg Obes Relat Dis. 2013;9:379-84.

104. Seki Y, et al. The effects of laparoscopic sleeve gastrectomy with duodenojejunal bypass on Japanese patients with BMI $<35 \mathrm{~kg} /$ $\mathrm{m}^{2}$ on type 2 diabetes mellitus and the prediction of successful glycemic control. Obes Surg. 2018;28:2429-38.

105. Seki Y, et al. Metabolic surgery for inadequately controlled type 2 diabetes in nonseverely obese Japanese: a prospective, singlecenter study. Surg Obes Relat Dis. 2018;14:978-85.

106. Lee $\mathrm{MH}$, et al. Laparoscopic sleeve gastrectomy for type 2 diabetes mellitus: long-term result and recurrence of diabetes. Obes Surg. 2020;30:3669-74.

107. Lee WJ, et al. Metabolic surgery for diabetes treatment: sleeve gastrectomy or gastric bypass? World J Surg. 2017;41:216-23.

108. Lee WJ, et al. Laparoscopic sleeve gastrectomy for type 2 diabetes mellitus: predicting the success by ABCD score. Surg Obes Relat Dis. 2015;11:991-6.

109. Still CD, et al. A probability score for preoperative prediction of type 2 diabetes remission following RYGB surgery. Lancet Diabetes Endocrinol. 2014;2:38-45.

110. Aron-Wisnewsky J, et al. The advanced-DiaRem score improves prediction of diabetes remission 1 year post Rouxen-Y gastric bypass. Diabetologia. 2017;60:1892-902.

111. Aminian A, et al. Individualized metabolic surgery score: procedure selection based on diabetes severity. Ann Surg. 2017;266:650-7.

112. Pucci A, et al. Type 2 diabetes remission 2 years post Roux-enY gastric bypass and sleeve gastrectomy: the role of the weight loss and comparison of DiaRem and DiaBetter scores. Diabetes Med. 2018;35:360-7.

113. Ohta M, et al. Prediction of long-term diabetes remission after metabolic surgery in obese East Asian patients: a comparison between ABCD and IMS scores. Obes Surg. 2021;31:1485-95.

114. Cotillard A, et al. Type 2 diabetes remission after gastric bypass: what is the best prediction tool for clinicians? Obes Surg. 2015;25:1128-32.
115. Lee WJ, et al. Preoperative prediction of type 2 diabetes remission after gastric bypass surgery: a comparison of DiaRem scores and ABCD scores. Obes Surg. 2016;26:2418-24.

116. Chen JC, et al. Prediction of type 2 diabetes remission after metabolic surgery: a comparison of the individualized metabolic surgery score and the ABCD score. Surg Obes Relat Dis. 2018;14:640-5.

117. Shen SC, et al. Validating risk prediction models of diabetes remission after sleeve gastrectomy. Obes Surg. 2019;29:221-9.

118. Ohta M, et al. Current status of laparoscopic bariatric/metabolic surgery in Japan: the sixth nationwide survey by the Japan Consortium of Obesity and Metabolic Surgery. Asian J Endosc Surg. 2021;14:170-7.

119. Ding L, et al. Comparative effectiveness of bariatric surgeries in patients with obesity and type 2 diabetes mellitus: a network meta-analysis of randomized controlled trials. Obes Rev. 2020;21: e13030.

120. Park $\mathrm{CH}$, et al. Comparative efficacy of bariatric surgery in the treatment of morbid obesity and diabetes mellitus: a systematic review and network meta-analysis. Obes Surg. 2019;29:2180-90.

121. Lee Y, et al. Laparoscopic sleeve gastrectomy versus laparoscopic Roux-en-Y gastric bypass: a systematic review and meta-analysis of weight loss, comorbidities, and biochemical outcomes from randomized controlled trials. Ann Surg. 2021;273:66-74.

122. Parrott $\mathbf{J}$, et al. Integrated health nutritional guidelines for the surgical weight loss patient-2016 update: micronutrients. Surg Obes Relat Dis. 2017;13:727-41.

123. Nicoletti CF, et al. New look at nutritional care for obese patient candidates for bariatric surgery. Surg Obes Relat Dis. 2013;9:520-5.

124. Jastrzębska-Mierzyńska M, et al. Assessment of dietary habits, nutritional status and blood biochemical parameters in patients prepared for bariatric surgery: a preliminary study. Wideochir Inne Tech Maloinwazyjne. 2012;7:156-65.

125. Lo Menzo E, et al. A nutritional implications of obesity: before and after bariatric surgery. Bariatr Surg Pract Patient Care. 2014;9:9-17.

126. Watanabe A, et al. Preoperative weight loss and operative outcome after laparoscopic sleeve gastrectomy. Obes Surg. 2017;27:2515-21.

127. Mechanick JI, et al. Clinical practice guidelines for the perioperative nutrition, metabolic, and nonsurgical support of patients undergoing bariatric procedures-2019 update: cosponsored by American Association of Clinical Endocrinologists/American College of Endocrinology, the Obesity Society, American Society for Metabolic \& Bariatric Surgery, Obesity Medicine Association, and American Society of AnesthesiologistsExecutive Summary. Endocr Pract. 2019;25:1346-59.

128. Collins J, et al. Preoperative weight loss in high-risk superobese bariatric patients: a computed tomographybased analysis. Surg Obes Relat Dis. 2011;7:480-5.

129. Fris RJ. Preoperative low energy diet diminishes liver size. Obes Surg. 2004;14:1165-70.

130. Colles SL, et al. Preoperative weight loss with a very-low-energy diet: quantitation of changes in liver and abdominal fat by serial imaging. Am J Clin Nutr. 2006;84:301-11.

131. Holderbaum M, et al. Effects of very low calorie diets on liver size and weight loss in the preoperative period of bariatric surgery: a systematic review. Surg Obes Relat Dis. 2018;14:237-44.

132. Wissen J, et al. Preoperative methods to reduce liver volume in bariatric surgery: a systematic review. Obes Surg. 2016;26:251-6. 
133. Alvarado R, et al. The impact of preoperative weight loss in patients undergoing laparoscopic roux-en-Y gastric bypass. Obes Surg. 2005; 15:1282-6.

134. Conaty EA, et al. Efficacy of a required preoperative weight loss program for patients undergoing bariatric surgery. J Gastrointest Surg. 2016;20:667-73 (in Japanese).

135. Japan Diabetes Society. Diabetes specialist training guidebook 8th ed. 2020. (in Japanese)

136. Japan Diabetes Society. Recommendations for the appropriate use of metformin. 2020. http://www.fa.kyorin.co.jp/jds/uploads/ recommendation_metformin.pdf. Accessed 15 Sept 2021

137. Dhatariya K, et al. NHS guideline for the perioperative management of the adult patient with diabetes. Diabetes Med. 2012;29:420-33.

138. Japan Diabetes Society. Recommendations for the appropriate use of SGLT2 inhibitor. 2020. http://www.fa.kyorin.co.jp/jds/ uploads/recommendation_SGLT2.pdf (in Japanese)

139. ASMBS Clinical Issues Committee. Peri-operative management of obstructive sleep apnea. Surg Obes Relat Dis. 2012;8:e27-32.

140. Hallowell PT, et al. Potentially life-threatening sleep apnea is unrecognized without aggressive evaluation. Am J Surg. 2007;193:364-7.

141. Frey WC, et al. Obstructive sleep-related breathing disorders in patients evaluated for bariatric surgery. Obes Surg. 2003;13:676-83.

142. Chung F, et al. Society of anesthesia and sleep medicine guidelines on preoperative screening and assessment of adult patients with obstructive sleep apnea. Anesth Analg. 2016;123:452-73.

143. Abdelsattar ZM, et al. The impact of untreated obstructive sleep apnea on cardiopulmonary complications in general and vascular surgery: a cohort study. Sleep. 2015;38:1205-10.

144. Coronavirus FAQs: CPAP tips for sleep apnea patients. American Academy of Sleep Medicine. 2020. https://aasm.org/coronaviruscovid-19-faqs-cpap-sleep-apnea-patients. Accessed 15 Sept 2021

145. James E, et al. Psychopathology before surgery in the Longitudinal Assessment of Bariatric Surgery-3(LABS3) Psychosocial Study. Surg Obes Relat Dis. 2012;8:533-41.

146. Luca MD, et al. Indications for surgery for obesity and weightrelated diseases: position statements from the international federation for the surgery of obesity and metabolic disorders (IFSO). Obes Surg. 2016;26:1659-96.

147. Brodsky JB, et al. Morbid obesity and tracheal intubation. Anesth Analg. 2002;94:732-6.

148. Langeron $\mathrm{O}$, et al. Prediction of difficult mask ventilation. Anesthesiology. 2000;92:1229-36.

149. Kheterpal S, et al. Incidence and predictors of difficult and impossible mask ventilation. Anesthesiology. 2006;105:885-91.

150. DeMaria EJ, et al. Validation of the obesity surgery mortality risk score in a multicenter study proves it stratifies mortality risk in patients undergoing gastric bypass for morbid obesity. Ann Surg. 2007;246:578-82.

151. Ogunnaike BO, et al. Anesthetic considerations for bariatric surgery. Anesth Analg. 2002;95:1793-805.

152. Schumann R. Anaesthesia for bariatric surgery. Best Pract Res Clin Anaesthesiol. 2011;25:83-93.

153. Owers CE, et al. Perioperative optimization of patients undergoing bariatric surgery. J Obes. 2012;2012:781546.

154. Buchwald $\mathrm{H}$, et al. Trends in mortality in bariatric surgery: a systematic review and meta-analysis. Surgery. 2007;142:621-32.

155. Greenstein AJ, et al. Prevalence of adverse intraoperative events during obesity surgery and their sequelae. J Am Coll Surg. 2012;215:271-7.

156. Stenberg E, et al. Early complications after laparoscopic gastric bypass surgery: results from the Scandinavian Obesity Surgery Registry. Ann Surg. 2014;260:1040-7.
157. Nelson DW, et al. Analysis of obesity-related outcomes and bariatric failure rates with the duodenal switch vs gastric bypass for morbid obesity. Arch Surg. 2012;147:847-54.

158. Hutter MM, et al. First report from the American College of Surgeons Bariatric Surgery Center Network: laparoscopic sleeve gastrectomy has morbidity and effectiveness positioned between the band and the bypass. Ann Surg. 2011;254:410-20.

159. Nguyen NT, et al. Laparoscopic versus open gastric bypass: a randomized study of outcomes, quality of life, and costs. Ann Surg. 2001;234:279-89.

160. Arterburn D, et al. Predictors of long-term mortality after bariatric surgery performed in Veterans Affairs medical centers. Arch Surg. 2009;144:914-20.

161. Melinek J, et al. Autopsy findings following gastric bypass surgery for morbid obesity. Arch Pathol Lab Med. 2002;126:1091-5.

162. Schauer $P$, et al. The learning curve for laparoscopic Roux-en-Y gastric bypass is 100 cases. Surg Endosc. 2003;17:212-5.

163. Wittgrove AC, et al. Laparoscopic gastric bypass, Roux-en-Y500 patients: technique and results, with 3-60 month follow-up. Obes Surg. 2000;10:233-9.

164. Schauer PR, et al. Outcomes after laparoscopic Roux-en-Y gastric bypass for morbid obesity. Ann Surg. 2000;232:515-29.

165. Westling A, et al. Laparoscopic vs open Roux-en-Y gastric bypass: a prospective, randomized trial. Obes Surg. 2001;11:284-92.

166. Nguyen NT, et al. A comparison study of laparoscopic versus open gastric bypass for morbid obesity. J Am Coll Surg. 2000;191:149-55.

167. Higa KD, et al. Complications of the laparoscopic Roux-en-Y gastric bypass: 1,040 patients-what have we learned? Obes Surg. 2000;10:509-13.

168. Papasavas PK, et al. Outcome analysis of laparoscopic Roux-enY gastric bypass for morbid obesity. The first 116 cases. Surg Endosc. 2002;16:1653-7.

169. Luján JA, et al. Laparoscopic gastric bypass in the treatment of morbid obesity. Preliminary results of a new technique. Surg Endosc. 2002;16:1658-62.

170. Mehran A, et al. Management of acute bleeding after laparoscopic Roux-en-Y gastric bypass. Obes Surg. 2003;13:842-7.

171. Nguyen NT, et al. Early gastrointestinal hemorrhage after laparoscopic gastric bypass. Obes Surg. 2003;13:62-5.

172. Lancaster RT, et al. Bands and bypasses: 30-day morbidity and mortality of bariatric surgical procedures as assessed by prospective, multi-center, risk-adjusted ACS-NSQIP data. Surg Endosc. 2008;22:2554-63.

173. Carlin AM, et al. The comparative effectiveness of sleeve gastrectomy, gastric bypass, and adjustable gastric banding procedures for the treatment of morbid obesity. Ann Surg. 2013;257:791-7.

174. Aurora AR, et al. Sleeve gastrectomy and the risk of leak: a systematic analysis of 4888 patients. Surg Endosc. 2012;26:1509-15.

175. Dick A, et al. Gastrointestinal bleeding after gastric bypass surgery: nuisance or catastrophe? Surg Obes Relat Dis. 2010;6:643-7.

176. Chapman A, Game P, O'Brien P, Maddern G, Kiroff G, Foster $\mathrm{B}$ et al. A systematic review oflaparoscopic adjustable gastric banding for the treatment of obesity (update and re-appraisal). Adelaide, SA: Australian Safety and Efficacy Register of New Interventional Procedures-Surgical(ASERNIP-S); 2002:31.

177. Buchwald $\mathrm{H}$, et al. Bariatric surgery: a systematic review and meta-analysis. JAMA. 2004;292:1724-37.

178. Stephens DJ, et al. Short-term outcomes for super-super obese $\left(\mathrm{BMi}>\right.$ or $\left.=60 \mathrm{~kg} / \mathrm{m}^{2}\right)$ patients undergoing weight loss surgery at a high-volume bariatric surgery center: laparoscopic adjustable gastric banding, laparoscopic gastric bypass, and open tubular gastric bypass. Surg Obes Relat Dis. 2008;4:408-15. 
179. Jones KB Jr, et al. Open versus laparoscopic Roux-en-Y gastric bypass: a comparative study of over 25,000 open cases and the major laparoscopic bariatric reported series. Obes Surg. 2006;16:721-7.

180. Longitudinal Assessment of Bariatric Surgery (LABS) Consortium, Flum DR, et al. Perioperative safety in the longitudinal assessment of bariatric surgery. N Engl J Med. 2009;361:445-54.

181. Tiwari MM, et al. Differences in outcomes of laparoscopic gastric bypass. Surg Obes Relat Dis. 2011;7:277-82.

182. Higgins RM, et al. Preoperative immobility significantly impacts the risk of postoperative complications in bariatric surgery patients. Surg Obes Relat Dis. 2018;14:842-8.

183. The Japanese Respiratory Society. Clinical Practice Guideline for Sleep Apnea Syndrome (SAS) 2020. Tokyo: Nankodo; 2020. (in Japanese)

184. Thorell A, et al. Guidelines for perioperative care in bariatric surgery: enhanced recovery after surgery (ERAS) society recommendations. World J Surg. 2016;40:2065-83.

185. Kikkawa E, et al. Surgical treatment for morbidly obese japanese patients: change in food intake, nutritional status and quality of life (QOL) one year after laparoscopic Roux-en-Y Gastric Bypass (LRYGB). J Jpn Society Study Obes. 2010;16:51-7 (in Japanese).

186. Kikkawa E, et al. Nutritional management and change in dietary intake after laparoscopic Roux-en-Y gastric bypass for the treatment of morbid obesity. J Jpn Society Study Clin Nutr. 2012;34:165-71 (in Japanese).

187. Kikkawa E, et al. Assessment of food satisfaction and food tolerance after bariatric surgery. J Jpn Soc Metab Clin Nutr. 2016;19:415-22 (in Japanese).

188. Kikkawa E, et al. Analysis of food satisfaction and tolerance after bariatric surgery in Japanese patients. J Jpn Soc Clin Nutr. 2015;36:10-5 (in Japanese).

189. Kikkawa E, et al. Dietary intake after bariatric surgery in Japanese patients with severe obesity and comparison between surgical techniques. J Jpn Soc Metab Clin Nutr. 2014;16:391-6 (in Japanese).

190. Hongo R, et al. Changes in nutrient intake and food preference before and after laparoscopic sleeve gastrectomy for patients with type 2 diabetes complicated severe obesity. Tonyobyo. 2019;62:143-54 (in Japanese)

191. Linda A, et al. ASMBS allied health nutritional guidelines for the surgical weight loss patient. Surg Obes Relat Dis. 2008;4:73-108.

192. Andreu A, et al. Protein intake, body composition, and protein status following bariatric surgery. Obes Surg. 2010;20:1509-15.

193. Saiki A, et al. The effects of partial use of formula diet on nutritional deficiency after bariatric surgery in severe obese patients: 12-month follow-up study. J Jpn Society Study Clin Nutr. 2014;36:1798-1805171 (in Japanese).

194. Kikkawa E, et al. Postoperative nutritional management and follow-up, metabolic surgery clinical update 135-140. Osaka: Medica Publishing; 2020. (in Japanese).

195. Caron M, et al. Long-term nutritional impact of sleeve gastrectomy. Surg Obes Relat Dis. 2017;13:1664-75.

196. Ministry of Health, Labour and Welfare. Dietary reference intakes for Japanese (2020). Tokyo: Daiichi syuppan; 2020. (in Japanese)

197. Wee CC, et al. High-risk alcohol use after weight loss surgery. Surg Obes Relat Dis. 2014;10:508-13.

198. Hood MM, et al. Managing severe obesity: understanding and improving treatment adherence in bariatric surgery. J Behav Med. 2016;39:1092-103.

199. Kushner R, et al. The American Board of Obesity Medicine: five year report. Obesity. 2017;25:982-3.

200. Dawes AJ, et al. Mental health conditions among patients seeking and undergoing bariatric surgery: a metaanalysis. JAMA. 2016;315:150-63.
201. Creel DB, et al. A randomized trial comparing two interventions to increase physical activity among patients under- going bariatric surgery. Obesity (Silver Spring). 2016;24:1660-8.

202. Courcoulas AP, et al. Weight change and health outcomes at 3 years after bariatric surgery among individuals with severe obesity. JAMA. 2013;310:2416-25.

203. Mulla CM, et al. Management of diabetes in patients undergoing bariatric surgery. Curr Diabetes Rep. 2019;19:112.

204. Aberle J, et al. Metformin after bariatric surgery: an acid problem. Exp Clin Endocrinol Diabetes. 2012;120:152-3.

205. Kashyap SR, et al. Double-blinded, randomized, and controlled study on the effects of canagliflozin after bariatric surgery: a pilot study. Obes Sci Pract. 2020;6:255-63.

206. Andalib A, et al. Diabetic ketoacidosis following bariatric surgery in patients with type2 diabetes. Diabetes Care. 2016;39:e121-122.

207. Capristo E, et al. Incidence of hypoglycemia after gastric bypass vs sleeve gastrectomy: a randomized trial. J Clin Endocrinol Metab. 2018;103:2136-46.

208. Sawada $\mathrm{S}$, et al. Continuous glucose monitoring in patients with remission of type 2 diabetes after laparoscopic sleeve gastrectomy without or with duodenojejunal bypass. Clin Obes. 2020;10: e12409.

209. Murphy R, et al. Progression of diabetic retinopathy after bariatric surgery. Diabet Med. 2015;32:1212-20.

210. Choi HK, et al. Bariatric surgery as urate-lowering therapy in severe obesity. Ann Rheum Dis. 2014;73:791-3.

211. Gadiraju S, et al. Levothyroxine dosing following bariatric surgery. Obes Surg. 2016;26:2538-42.

212. Pellitero S, et al. Evaluation of vitamin and trace element requirements after sleeve gastrectomy at long term. Obes Surg. 2017;27:1674-82.

213. Kwon Y, et al. Anemia, iron and vitamin B12 deficiencies after sleeve gastrectomy compared to Roux-en-Y gastric bypass: a metaanalysis. Surg Obes Relat Dis. 2014;10:589-97.

214. Cummings DE, et al. Metabolic surgery for the treatment of type 2 diabetes in obese individuals. Diabetologia. 2018;61:257-64.

215. Sarkhosh K, et al. The impact of bariatric surgery on obstructive sleep apnea: a systematic review. Obes Surg. 2013;23:414-23.

216. Dixon JB, et al. Surgical vs conventional therapy for weight loss treatment of obstructive sleep apnea: a randomized controlled trial. JAMA. 2012;308:1142-9.

217. Seki Y, et al. Prevalence of nonalcoholic steatohepatitis in Japanese patients with morbid obesity undergoing bariatric surgery. J Gastroenterol. 2016;51:281-9.

218. Nikai H, et al. Effects of laparoscopic sleeve gastrectomy on nonalcoholic steatohepatitis and liver fibrosis in Japanese patients with severe obesity. Obes Surg. 2020;30:2579-87.

219. Coupaye M, et al. Comparison of the incidence of cholelithiasis after sleeve gastrectomy and Roux-en-Y gastric bypass in obese patients: a prospective study. Surg Obes Relat Dis. 2015;11:779-84.

220. Mihmanli M, et al. Effects of laparoscopic sleeve gastrectomy on parathyroid hormone, vitamin $\mathrm{D}$, calcium, phosphorus, and albumin levels. Obes Surg. 2017;27:3149-55.

221. Hewitt $\mathrm{S}$, et al. Relationships between vitamin D status and PTH over 5 years after Roux-en-Y gastric bypass: a longitudinal cohort study. Obes Surg. 2020;30:3426-34.

222. Bariatric Support Centers International: BSCI Certified Support Group Leader Guidebook 21-25. Utah: Bariatric Support Centers International; 2012.

223. Hayashi K. Surgical treatment corner. Points to note before and after bariatric and metabolic surgery from the perspective of a mental health professional. J Jap Soc Treat Obes. 2019;7:20-1 (in Japanese).

224. Conason A, et al. Substance use following bariatric weight loss surgery. JAMA Surg. 2013;148:145-50. 
225. Bhatti JA, et al. Self-harm emergencies after bariatric surgery: a population-based cohort study. JAMA Surg. 2016;151:226-32.

226. Peterhansel C, et al. Risk of completed suicide after bariatric surgery: a systematic review. Obes Rev. 2013;14:369-82.

227. Castaneda D, et al. Risk of Suicide and Self-harm Is Increased After Bariatric Surgery-a Systematic Review and Meta-analysis. Obes Surg. 2019;29:322-33.

228. Jakobsen GS, et al. Association of bariatric surgery vs medical obesity treatment with long-term medical complications and obesityrelated comorbidities. JAMA. 2018;319:291-301.

229. Pizato N, et al. Effect of Grazing behavior on weight regain postbariatric surgery: a systematic review. Nutrients. 2017;9:1322.

230. Szmulewicz A, et al. Mental health quality of life after bariatric surgery: A systematic review and meta-analysis of randomized clinical trials. Clinical Obesity. 2019;9: e12290.

231. Tindle HA, et al. Risk of suicide after long-term follow-up from bariatric surgery. Am J Med. 2010;123:1036-42.

232. Bellicha A, et al. Effective of exercise training after bariatric surgery: a systematic literature review and meta-analysis. Obes Rev. 2018;19:1544-56.

233. Daniels $P$, et al. Effect of a randomised 12-week resistance training program on muscular strength, cross- sec-tional area and muscle quality in women having undergone Roux-en-Y gastric bypass. J Sports Sci. 2018;36:529-35.

234. Baillot A, et al. Effects of a pre-surgery supervised exercise training 1 year after bariatric surgery: a randomized controlled study. Obes Surg. 2018;28:955-62.

235. Tabesh MR, et al. Nutrition, physical activity, and prescription of supplements in pre- and post-bariatric surgery patients: a practical guideline. Obes Surg. 2019;29:3385-400.

236. Nishijima M, et al. Obstructive sleep apnea syndrome associated with severe obesity(preoperative evaluation and management) Metabolic Surgery Clinical Update 66-71. Osaka: Medica Publishing; 2020. (in Japanese).

237. Kikkawa E, et al. Effect of dietary habits after bariatric surgery on the effect of weight loss. J Jpn Soc Clin Nutr. 2019;41:128-34 (in Japanese)

Publisher's Note Springer Nature remains neutral with regard to jurisdictional claims in published maps and institutional affiliations.

\section{Authors and Affiliations}

\section{Akira Sasaki ${ }^{1}$ (1) $\cdot$ Koutaro Yokote $^{2} \cdot$ Takeshi Naitoh $^{3} \cdot$ Junji Fujikura $^{4} \cdot$ Karin Hayashi $^{5} \cdot$ Yushi Hirota $^{6}$. Nobuya Inagaki ${ }^{4} \cdot$ Yasushi Ishigaki $^{7} \cdot$ Kazunori Kasama $^{8} \cdot$ Eri Kikkawa $^{8} \cdot$ Hidenori Koyama $^{9} \cdot$ Hiroaki Masuzaki $^{10}$. Takeshi Miyatsuka ${ }^{11} \cdot$ Takehiro Nozaki $^{12}$. Wataru Ogawa ${ }^{6} \cdot$ Masayuki Ohta $^{13} \cdot$ Shinichi Okazumi $^{14}$. Michio Shimabukuro ${ }^{15}$ - lichiro Shimomura ${ }^{16}$. Hitoshi Nishizawa ${ }^{16}$. Atsuhito Saiki ${ }^{17}$. Yosuke Seki ${ }^{8}$. Nobuhiro Shojima ${ }^{18} \cdot$ Motoyoshi Tsujino $^{19} \cdot$ Satoshi Ugi ${ }^{20}$. Hiroaki Watada ${ }^{11}$. Toshimasa Yamauchi ${ }^{18}$. Takashi Yamaguchi $^{17} \cdot$ Kohjiro Ueki $^{21} \cdot$ Takashi Kadowaki $^{22} \cdot$ Ichiro Tatsuno $^{23} \cdot$ Joint Committee in the Japanese Society for Treatment of Obesity, the Japan Diabetes Society, the Japan Society for the Study of Obesity}

1 Department of Surgery, Iwate Medical University, 2-1-1 Idaidori, Yahaba 028-3695, Japan

2 Department of Endocrinology Hematology, and Gerontology, Graduate School of Medicine, Chiba University, Chiba, Japan

3 Department of Lower Gastrointestinal Surgery, Kitasato University School of Medicine, Sagamihara, Japan

4 Department of Diabetes, Endocrinology and Nutrition, Kyoto University Graduate School of Medicine, Kyoto, Japan

5 Department of Neuropsychiatry, Toho University Sakura Medical Center, Sakura, Chiba, Japan

6 Division of Diabetes and Endocrinology, Kobe University Graduate School of Medicine, Kobe, Japan

7 Division of Diabetes and Metabolism, Department of Internal Medicine, Iwate Medical University, Yahaba, Japan

8 Weight loss and Metabolic Surgery Center, Yotsuya Medical Cube, Tokyo, Japan

9 Department of Diabetes, Endocrinology and Clinical Immunology, Hyogo College of Medicine, Nishinomiya, Hyogo, Japan

10 Division of Endocrinology, Diabetes and Metabolism, Hematology, Rheumatology, Department of Medicine, Graduate School of Medicine, University of the Ryukyus, Okinawa, Japan
11 Department of Metabolism and Endocrinology, Juntendo University Graduate School of Medicine, Tokyo, Japan

12 Clinical Trial Center, Kyushu Central Hospital of the Mutual Aid Association of Public School Teachers, Fukuoka, Japan

13 Global Oita Medical Advanced Research Center for Health, Oita University, Oita, Japan

14 Department of Surgery, Toho University Sakura Medical Center, Sakura, Chiba, Japan

15 Department of Diabetes, Endocrinology and Metabolism, School of Medicine, Fukushima Medical University, Fukushima, Japan

16 Department of Metabolic Medicine, Graduate School of Medicine, Osaka University, Osaka, Japan

17 Center of Diabetes, Endocrine and Metabolism, Toho University Sakura Medical Center, Sakura, Japan

18 Department of Diabetes and Metabolic Diseases, Graduate School of Medicine, The University of Tokyo, Tokyo, Japan

19 Department of Endocrinology and Metabolism, Tokyo Metropolitan Tama Medical Center, Tokyo, Japan

20 Division of Endocrinology and Metabolism, Department of Medicine, Shiga University of Medical Science, Otsu, Japan 
21 Department of Molecular Diabetic Medicine, Diabetes Research Center, National Center for Global Health and Medicine, Tokyo, Japan

22 Toranomon Hospital, Tokyo, Japan
23 Chiba Prefectural University of Health Sciences, Chiba, Japan 